\title{
Comparing syngeneic
}

and autochthonous models of breast cancer to identify tumor immune components that correlate with response to immunotherapy in breast cancer

Jessica Castrillon Lal

Dana Farber Cancer Institute

Anita K. Mehta

Dana Farber Cancer Institute

Madeline G. Townsend

Brigham and Women's Hospital

\section{Madisson Oliwa}

Brigham and Women's Hospital

Eric Miller

NanoString Technologies Inc

Alaba Sotayo

Dana Farber Cancer Institute

Emily Cheney

Dana Farber Cancer Institute

Elizabeth A. Mittendorf

Dana Farber Cancer Institute

Anthony Letai

Dana Farber Cancer Institute

Jennifer L. Guerriero ( $\square$ Jennifer_Guerriero@dfci.harvard.edu )

Dana Farber Cancer Institute https://orcid.org/0000-0002-2104-5457

Research article

Keywords: breast cancer, immunotherapy, immune checkpoint blockade, syngeneic tumor models, preclinical mouse models

Posted Date: January 28th, 2021

DOl: https://doi.org/10.21203/rs.3.rs-57679/v2 
License: (c) (i) This work is licensed under a Creative Commons Attribution 4.0 International License. Read Full License

Version of Record: A version of this preprint was published at Breast Cancer Research on August 5th, 2021. See the published version at https://doi.org/10.1186/s13058-021-01448-1. 


\section{Abstract}

Background: The heterogeneity of the breast tumor microenvironment (TME) may contribute to the lack of durable responses to immune checkpoint blockade (ICB), however, mouse models to test this are currently lacking. Proper selection and use of preclinical models are necessary for rigorous, preclinical studies to rapidly move laboratory findings into the clinic to treat patients.

Methods: We compared 3 versions of a common syngeneic and autochthonous mouse model to elucidate how tumor latency and TME heterogeneity contributes to ICB resistance. We performed comprehensive characterization of the TME using quantitative flow-cytometry and RNA expression analysis (NanoString) utilizing three distinct syngeneic breast cancer models, all derived from the MMTVPyMT autochthonous model. A commonly used protocol was used to obtain tumor cells from MMTVPyMT mice and 1E6, 1E5 or 1E4 cells were immediately injected into the mammary fat pad of FVB/NJ wild type mice. We then performed deep immunophenotyping and tested ICB efficacy in the 3 syngeneic models compared to the autochthonous model.

Results: The 4 models had vastly different TMEs that correlated to ICB responses. We found that the number of cells used to generate syngeneic tumors significantly influences tumor latency, infiltrating leukocyte population and response to ICB. Compared to the autochthonous model, all 3 syngeneic models had significantly more tumor infiltrating lymphocytes (TILs; $C D 3^{+}, \mathrm{CD} 4^{+}$, and $\mathrm{CD} 8^{+}$) and higher proportions of PD-L1 positive myeloid cells, whereas the MMTV-PyMT model had the highest frequency of myeloid cells out of total leukocytes. Increased TILs correlated with response to anti-PD-L1 and antiCTLA-4 therapy; but PD-L1 expression on tumor cells or PD-1 expression of T-cells did not.

Conclusions: These studies reveal that the commonly used syngeneic models have low concordance with the autochthonous model. We have identified ICB-sensitive and resistant syngeneic breast cancer models, generated from the same tumor cell inoculum, and find that only the 1E4 syngeneic model is representative of the slow growing, autochthonous model. Given the lack of benefit from ICB in breast cancer, the identification of robust murine models presented here provides the opportunity to further interrogate the TME for breast cancer treatment and provide novel insights into therapeutic combinations to overcome ICB resistance.

\section{Background}

The success of immune checkpoint blockade (ICB) in a variety of human cancer types has stimulated interest in its use for treating breast cancer. Current therapy for breast cancer is guided by the molecular pathology of the tumor. Breast cancer is often driven by overactive hormone signaling (estrogen and/or progesterone receptors; ER, PR) or amplification of growth factor response (HER2) and therefore treated with endocrine therapy or HER2-targeted agents. Alternatively patients can also be treated with general therapies such as chemotherapy and/or radiation. ${ }^{1-4}$ After initial treatment for early stage disease, approximately $30 \%$ of women will eventually develop recurrent advanced or metastatic disease. ${ }^{5}$ Almost 
all who develop metastatic breast cancer will succumb to the disease, highlighting the need for more effective strategies. ${ }^{6}$

Advances in understanding tumor-host immune interactions and their role in cancer progression have led to novel therapeutic strategies for cancer. ICB aims to target T-cell inhibitory molecules using antibodies against cytotoxic T-lymphocyte associated protein 4 (CTLA-4) and programmed cell death protein-1 (PD1 ) as well as its ligand (PD-L1) which play an important role in central and peripheral immune tolerance. ICB reinvigorating anti-tumor immune responses by inhibiting negative interactions between T-cells and antigen presenting cells (APCs) or tumor cells in several cancer types. ${ }^{7,8}$ In 2011, the first ICB agent, ipilimumab, a human monoclonal antibody targeting CTLA-4, was approved by the FDA for treatment of metastatic melanoma based on significant improvement in overall survival in a randomized, doubleblinded Phase III study. ${ }^{9}$ Importantly, ipilimumab has doubled 10-year survival for metastatic melanoma compared with historical data. ${ }^{10-12}$ Antibodies targeting PD-1 and PD-L1 have also shown a durable clinical response in melanoma, as well as renal cell carcinoma, non-small cell lung cancer, and bladder cancer. ${ }^{13-20}$ To date, such responses have led to FDA approval of seven ICB therapies for the treatment of more than 15 different types of cancer. ${ }^{21}$ Because their effector pathways are distinct, the combination of CTLA-4 and PD-1/PD-L1 therapy can provide an enhanced response. ${ }^{22,23}$ The combination has been FDA approved for melanoma ${ }^{24}$, renal cell carcinoma ${ }^{25}$, non-small cell lung cancer ${ }^{26}$ and colorectal cancer. ${ }^{27}$

Breast cancer has a lower mutational burden compared to other types of cancer which may explain the lack of efficacy in response to ICB. ${ }^{28,29}$ Despite this generalization, triple negative breast cancer (TNBC) has demonstrated some benefit from ICB therapy, albeit not achieving the response rates demonstrated in melanoma and lung cancer. While pembrolizumab (anti-PD-1) showed promising activity as a single agent against advanced or metastatic TNBC in the KEYNOTE-012 (NCT01848834) and KEYNOTE-086 (NCT02447003) clinical trials, in the randomized, Phase III KEYNOTE-119 (NCT02555657) clinical trial there was no improvement in overall survival compared to single-agent chemotherapy in metastatic TNBC. ${ }^{30}$ Benefit from ICB therapy has been observed in patients treated in the first line setting and/or in patients whose tumors or immune cells express PD-L1. ${ }^{31-33}$ For example, in the PCD4989g (NCT01375842) clinical trial, evaluating atezolizumab as a single agent, expression of PD-L1 on $1 \%$ or greater of immune infiltrating cells was associated with a $12 \%$ ORR compared to $0 \%$ when there was no expression of PD-L1, and high levels of immune cell infiltration (greater than 10\%) was independently associated with higher overall response rate (ORR) and overall survival (OS).$^{32} \mathrm{Importantly}$, there are two FDA approved ICB therapies for breast cancer, the first that came from the Phase III IMpassion130 (NCT02425891) clinical trial that demonstrated atezolizumab in combination with nab-paclitaxel showed significant extension of median disease-free overall survival compared to nab-paclitaxel alone, from 15.5 to 25 months in patients with $1 \%$ or more PD-L1 positive immune cells, where there was no benefit in PDL1 negative tumors. ${ }^{34,35}$ More recently, pembrolizumab, an anti-PD-1 antibody, in combination with different chemotherapy agents, was also approved for the treatment of locally advanced or metastatic TNBC, based on results from the KEYNOTE-355 trial. ${ }^{36}$ 
The use of PD-L1 as a biomarker for ICB has been rigorously investigated but has raised concerns including poor agreement between different antibodies as well as scoring between pathologists. ${ }^{37}$ To date, there are 9 FDA approvals for the use of ICB based on a specific PD-L1 threshold and companion diagnostic, with variable thresholds both within and across tumor types using several different assays, including approvals at the following PD-L1 positive percentage thresholds: 1, 5, and 50\%. In a recent meta-analysis that examined all approvals of ICB as of April 2019, PD-L1 was predictive in $28.9 \%$ of those approvals, and was either not predictive $(53.3 \%)$ or not tested $(17.8 \%)$ in the remaining approvals. ${ }^{21}$ This underscores the need to improve ICV efficacy biomarkers and assess what cells expressing PD-L1 are the most predictive of response. Other predictors of response to ICB include the presence of immune cells. In HER2 ${ }^{+}$and TNBC tumors, immune cells have been shown to correlate with better response to HER2-targeted therapy and chemotherapy, respectively. ${ }^{38}$ However, immune cell infiltration has been reported to differ among each subtype of breast cancer. ${ }^{39}$ Further work to characterize the TME of breast tumors will provide more opportunities for ICB therapy in these patients.

Mouse models have been instrumental in understanding the molecular mechanisms of oncogenesis and metastasis. Being able to translate in vivo preclinical findings to patients depends on how accurately the mouse model replicates histological markers, biochemical pathways, and genetic aberrations observed in the same human tumor type. ${ }^{40}$ In light of the advances in immunotherapy, it is now also necessary to meticulously characterize the TME of preclinical mouse models. Here we have generated and characterized 3 models of breast cancer, derived from a commonly used autochthonous model of breast cancer, in which the polyoma middle T (PyMT) oncogene is driven by the mouse mammary tumor virus (MMTV)-LTR. The MMTV-PyMT model is representative of human breast carcinomas; where several of the same signaling transduction pathways that are commonly disrupted in human breast cancer patients are seen in the MMTV-PyMT model such as the Src family, Ras and PI3K kinase pathways. ${ }^{41,42}$ In addition, both innate and adaptive immune cells infiltrate the tumor during tumorigenesis. ${ }^{43,44}$ Macrophages have been shown to play a key role in the development of these tumors, in which CCL2 recruits inflammatory monocytes to facilitate breast tumor growth and metastasis. ${ }^{45}$ Additionally, it has been shown that the phenotype is mediated through IL-4 expressing CD $4^{+}$T-cells. ${ }^{44} \mathrm{~A}$ spectrum of macrophage phenotypes have been recognized, ranging from classically activated macrophages ("M1"like) that are effective in clearing intracellular pathogens and can recruit cytotoxic $T$ lymphocytes to activate adaptive immune responses ${ }^{46}$; to alternatively activated macrophages ("M2"-like), which function to help with parasite clearance, exhibit tissue remodeling capabilities and promote tumor progression by recruiting $\mathrm{T}$ regulatory and Th2 T-cell subsets lacking cytotoxic functions. ${ }^{47}$ Tumor associated macrophages (TAMs) likely exhibit features of both M1- and M2-like macrophages but in general, exhibit an M2-like phenotype that promotes tumor progression and metastasis by secreting factors that regulate angiogenesis and recruit tumor suppressive cells such as $T$ regulatory (Treg) cells. ${ }^{48,49}$ In line with the lack of clinical efficacy of ICB in breast cancer, preclinical studies have shown that MMTV-PyMT mice are resistant to ICB monotherapy. ${ }^{43,50,51}$ 
With respect to ICB therapy in the clinical care of breast cancer patients, work is urgently needed to better understand if immune cell infiltration, including type, number and/or phenotype, correlates to responses. Understanding what factors are critical for ICB efficacy in breast cancer will allow careful patient selection and/or catalyze clinical development of novel therapies to distinguish non-responders to responders, improve responses that do occur, surmount acquired resistance to immunotherapy and identify biomarkers that can more accurately predict durable response. Using the right preclinical model is challenging. While the autochthonous MMTV-PyMT best represents the human disease $\mathrm{s}^{41,52,53}$, there are significant financial and time constraints to using this model. Therefore, researchers have adopted the use of transplanting MMTV-PyMT tumor cells into wild-type mice. With translation to the clinic being of high priority, we sought to determine how the autochthonous and derived syngeneic models align. To accomplish this, we generated three distinct syngeneic models derived from the MMTV-PyMT model and performed deep immuno-phenotypic analysis and ICB efficacy. We identify poor concordance between the 1E6 and 1E5 syngeneic models with the autochthonous model. In addition, we identify biomarkers and immune mechanisms that correlate with response to ICB therapy.

\section{Methods}

\section{Animal husbandry}

All experiments used either virgin female FVB/NJ mice or virgin female FVB/N autochthonous mice carrying the polyoma middle T (PyMT) transgene under the control of the mammary tumor virus (MMTV) promoter. The FVB/NJ (001800) and Nude (NU/J; 002448) mice were purchased from Jackson laboratory. All mice were maintained within the Dana-Farer Cancer Center (DFCI) and all experiments were conducted under The Institutional Animal Care and Use Committee (IACUC).

\section{Generation of syngeneic models}

For each experiment, we harvested separate batches of tumors from autochthonous MMTV-PyMT mice (referred to as "inoculum"). Using an established protocol, that is widely used ${ }^{54-57}$, late stage MMTVPyMT tumors were harvested and tumor suspension was either immediately injected into recipient FVB/NJ wild-type mice or frozen for subsequent experiments. Each batch of inoculum was simultaneously injected into wildtype mice using one million (1E6), one hundred thousand (1E5) or ten thousand (1E4) tumor cells. Each experiment was run using the same batch of inoculum, with 3-7 recipient mice per group. This entire protocol was repeated 3 times, starting with a new batch of inoculum for each experiment. The tumor suspension was never cultured. Each experiment was performed with 2-3 different batches of cells harvested from MMTV-PyMT mice. FVBN/J or Nude mice were inoculated with one million (1E6), one hundred thousand (1E5), or ten thousand (1E4) cells in the $4^{\text {th }}$ mammary fat pad to generate syngeneic models. The wild-type mice were age matched to the autochthonous mice at 10-12 weeks of age.

\section{Tumor digestion}


Tumors were extracted and minced, and subsequently blended using the gentleMACS Dissociator (Miltenyi Biotec cat. \#130-093-235). MACS Miltenyi Tumor Dissociation Kit for mouse (Miltenyi Biotec cat. \#130-096-730) was used for further enzymatic digestion according to the manufacturer's protocol. Dissociated tumor cell suspensions were rinsed with RPMI Medium 1640 (Life Technologies cat. \# 11875-093), filtered using a $70 \mu \mathrm{m}$ sterile EASY-strainer ${ }^{\mathrm{TM}}$ (Greiner bio-one cat. \#542 070) and performed red blood cell lysis using RBC Lysis Solution (Qiagen cat. \#158904). The Mouse Tumor Cell Isolation Kit (Miltenyi Biotec: 130-110-187) was used to remove $\mathrm{CD} 45^{+}$cells from the inoculum using the Miltenyi Automacs following standard procedures.

\section{Efficacy studies}

Caliper measurements were used to calculate tumor volumes for each mammary tumor using [(Length $\mathrm{x}$ Width $\left.{ }^{2}\right) / 2$ ]. MMTV-PyMT mice were enrolled into as study at about 80 days old and when each tumor reached $80-100 \mathrm{~mm}^{3}$. Tumors from mammary fat pad numbers 5 and 10 were excluded from the analysis. The sum of the volumes for the MMTV-PyMT autochthonous tumors (1-4 and 5-9) were used and indicated as "total tumor burden". Syngeneic mice that had tumor measurements ranging between 80-100 $\mathrm{mm}^{3}$ were enrolled into an experiment. At the indicated time points animals were euthanized in a $\mathrm{CO}_{2}$ chamber before performing a cardiac perfusion with normal saline. Lungs and tumors were removed for analysis.

\section{Flow cytometry}

Tumors were digested as described above, and single cells were re-suspended in a buffer containing $2 \%$ FBS and 2mM EDTA (Sigma-Aldrich cat. \#E7889) diluted in Phosphate buffered saline (PBS) (Lift Technologies cat. \#10010-023). Zombie Aqua ${ }^{\mathrm{TM}}$ Fixable Viability Kit (BioLegend cat. \#423101) and antimouse CD16/CD32 Fc gamma receptor II/III blocking antibody (Affymetrix cat. \#14-0161) were diluted in PBS and applied to cells on ice for 20 minutes in the dark on ice. Cells were washed and incubated with fluorochrome-conjugated antibodies (anti-mouse CD45 Alexa Fluor ${ }^{\circledR} 488$, clone 30-F11, BioLegend cat. \#103122; anti-mouse CD11b Brilliant Violet 711, clone M1/70, BioLegend cat. \#101241; anti-mouse CD3 Alexa Fluor ${ }^{\circledR}$ 594, clone 17A2, BioLegend cat. \#100240; anti-mouse MHCII Brilliant Violet 421, clone M5/114.15.2, BioLegend cat. \#107631; anti-mouse F4/80 Alexa Fluor ${ }^{\circledR} 647$, clone BM8, BioLegend cat. \#123122; anti-mouse CD11c Brilliant Violet 650, clone HL3, BD Biosciences cat. 564079; anti-mouse CD80 Brilliant Violet 605, clone 16-10A1, BioLegend cat. \#104729; anti-mouse CD86 PerCP/Cy5.5, clone GL-1, BioLegend cat. \#105027; anti-mouse CD40 PE/Cy7, clone 3/23, BioLegend cat. \#124621; antimouse CD206 PE, clone C068C2, BioLegend cat. \#141706; anti-mouse CD8 PE/Cy7, clone 53-6.7, BioLegend cat. \#100721; anti-mouse CD4 PE, clone GK1.5, BioLegend cat. \#100408; anti-mouse Ly6G/Ly-6C (GR1) Brilliant Violet 650, clone RB6-8C5, BioLegend cat. \#108441; anti-mouse PD-1 Brilliant Violet 421, clone 29F.1A12, BioLegend cat. \#135217; anti-mouse PDL-1 PE, clone 10F.9G2, BioLegend cat. \#124307, anti-mouse CD-19 Brilliant Violet 605, clone 6D5, BioLegend cat \#115539, anti-mouse NK1.1 Alexa Fluor® 647, clone PK136, BioLegend cat. \#108719, anti-mouse CD31 Brilliant Violet 421, clone 390, BioLegend cat. \#102423, anti-mouse Thy1.1 Brilliant Violet 650, clone OX-7, BioLegend cat. \#202533, 
anti-mouse Thy1.2 PerCP/Cy5.5, clone 53-2.1, BioLegend cat. \#140321) in the dark for an hour usingt the dilution recommended by the manufacturer. Following staining, cells were rinsed with PBS buffer and fixed with $1 \%$ paraformaldehyde for 5 minutes at room temperature. Afterward, cells were rinsed with PBS, re-suspended in PBS buffer, and placed in the dark at $4^{\circ} \mathrm{C}$ until analysis. Following extracellular staining, cells that obtained an intracellular stain were washed, fixed, and permeabilized using the Foxp3/ Transcription Factor Staining Buffer Set Kit (Affymetrix cat. \#00-5523) according to the manufacturer's protocol. Cells were incubated with antibody (anti-mouse Granzyme B Alexa Fluor ${ }^{\circledR}$ 647, clone GB11, BioLegend cat. \#515405; anti-mouse FoxP3 PerCP/C5.5, clone R16-715 BD, Biosciences cat. \#563902) overnight in the dark at $4^{\circ} \mathrm{C}$. The following day, cells were rinsed with PBS, and re-suspended with PBS buffer for flow cytometric analysis on the BD LSRFortessa at the Hematologic Neoplasia Flow Cytometry Core of the Dana-Farber Cancer Institute. Five hundred thousand to two million cells were analyzed per sample per mouse using BD FACs Diva Software. Single color controls were included in the quality control analysis. Total number of mice used per experiment are shown in each graph $(1 \mathrm{E} 6=15,1 \mathrm{E} 5=17$, $1 E 4=14, T g=16)$. In two instances there was one less sample due to technical or experimental error and 1 mouse's weight was not recorded in the $1 \mathrm{E} 6$ group and therefore enumeration calculations could not be performed and one tumor sample in the $1 \mathrm{E} 5$ group failed on flow cytometry in the intracellular staining panel. Otherwise all samples were used for analysis. Data analysis and compensation were performed on BD FACS Diva software. The absolute cell number populations were calculated using the equation below:

$$
\text { absolute cell population }=\frac{\% \text { cell population }}{\text { viable } * \mid \text { viable cells } \mid}
$$

Student's t-tests were performed in Prism version 7 (Graphpad, Inc.), and P values are designated as * $\mathrm{P}<$ $0.05,{ }^{\star \star} P<0.01,{ }^{\star \star \star} P<0.001$. All graphs show mean and error bars represent standard error of the mean (s.e.m).

\section{Dosing}

All in vivo experiments were treated with intraperitoneal injections. Mice were treated twice a week with 200 ug of InVivoMAb rat IgG2b isotype control, anti-keyhole limpet hemocyanin (clone LTF-2, BioXcell BE0090), InVivoMab anti-mouse CTLA-4 (clone 9H10, BioXcell BE 0131), and InVivoMab anti-mouse PDL1 (clone 10F9G2, BioXcell BE0101). Mice were treated until tumors reached $2 \mathrm{~cm}$ in one direction. Mouse weight was monitored and recorded weekly. Tumor volumes were measured and plotted as mean total tumor burden \pm SEM. Significant differences in tumor fold change were measured by a two-way analysis of variance (ANOVA) multiple comparisons on ranks. The statistical significance of survival curves was assessed using the Kaplan-Meier log rank analysis. All statistical analysis was performed in Prism version 7 (Graphpad, Inc.). $P$ values are designated as * $P<0.05$, $* * P<0.01$, $* \star * ~ P<0.001$.

\section{RNA isolation}


When syngeneic mouse tumors reached $100 \mathrm{~mm}^{3}$, tissue samples were snap frozen for later processing. Samples were also collected from autochthonous mice with total tumor burden in the range of 300-600 $\mathrm{mm}^{3}$. Tumor specimens of $30 \mathrm{mg}$ were used for RNA isolation using the RNeasy Mini Kit (Qiagen cat. \#74104). $\beta$-Mercaptoethanol was added to Buffer RLT and subsequently added to each tumor sample. Tissue was disrupted and homogenized using a 20 -gauge needle. An equal volume of $70 \%$ ethanol was added, transferred to a RNeasy spin column and centrifuged for 30 seconds at 12,000 rpm. The flowthrough was discarded. Buffer RW1 was added to the RNeasy spin column and centrifuged at 12,000 rpm for 30 seconds. The flow-through was discarded. Residual DNA was removed using the RNase-Free DNase Set (Qiagen cat. \#79254) according to the manufacturer's protocol. RPE was added to the RNeasy spin column and centrifuged at 12,000 rpm for 30 seconds. This step was repeated and centrifuged at the same speed for 2 minutes. RNeasy spin column was placed in a clean $2 \mathrm{ml}$ collection tube. Samples were eluted with $50 \mathrm{ul}$ of RNase-free water for 1 minute at 12,000 rpm. Samples were analyzed by the nanodrop to detect concentration and 260/230 ratio. RNA purity was assessed using the Agilent Bioanalyzer 4200 at the Molecular Biology Core Facility of Dana-Farber Cancer Institute.

\section{Immune profile gene analysis}

Purified RNA was isolated from murine tumors. Isolated RNA was submitted to the Center for Advanced Molecular Diagnostics core facility at Brigham and Women's Hospital. Gene expression analysis was conducted using the nCounter PanCancer Immune Profiling panel which includes 770 immune-related genes and relevant controls. NanoString gene expression values were normalized using the best subset of the 40 reference genes included in the panel, as determined by the geNorm algorithm ${ }^{58}$. The nSolver Advanced Analysis 2.0 software was used to perform all normalization. Pathway signatures were calculated by condensing biologically related groups of genes using the first principal component of their expression data. ${ }^{59}$ Cell type scores were calculated using the average log2 normalized expression of each cell type's marker genes. The cell type abundance scoring is modified from other reports ${ }^{60}$ where strict cell type gene correlation-driven QC $\mathrm{p}$-values were determined based on data that passed QC.

\section{Results}

\section{Tumor infiltrating leukocyte populations are significantly altered between the 4 murine breast cancer models.}

Three distinct preclinical models of breast cancer were generated by isolating tumors from autochthonous MMTV-PyMT mice, dissociating the tumors into single cell suspensions, referred to as inoculum, and directly inoculating $1 \mathrm{E} 6,1 \mathrm{E} 5$ or $1 \mathrm{E} 4$ cells into the $4^{\text {th }}$ mammary fat pad of wild-type FVB/NJ mice (Fig. 1a). Flow cytometry was used to assess the composition of cells in the inoculum that were injected into recipient mice (gating strategy in Supplemental Fig. 1a), which revealed that the cell suspension was composed of approximately $15 \% \mathrm{CD} 45^{+}$and $85 \% \mathrm{CD} 45^{-}$cells (Fig. $1 \mathrm{~b}$ ). The $\mathrm{CD} 45^{+}$cells represented myeloid cells $\left(\mathrm{CD} 11 \mathrm{~b}^{+}, \mathrm{CD} 11 \mathrm{c}^{+}, \mathrm{F} 4 / 80^{+}\right)$, T cells $\left(\mathrm{CD} 3^{+}\right)$, B cells $\left(\mathrm{CD} 19^{+}\right)$and NK cells $\left(\mathrm{NK} 1.1^{+}\right.$; 
Supl. Fig. 1b) and the CD45 cells consisted of tumor cells as well as a small percent of both endothelial cells $\left(\mathrm{CD} 31^{+}\right)$and fibroblasts (Thy $1.1^{+}$and Thy $1.2^{+}$; Fig. 1b). Tumor latency was directly related to the number of cells that were inoculated into the mammary fat pad, as inoculating fewer cells correlated with a longer time to reach $100 \mathrm{~mm}^{3}$ (Fig. 1C). It took approximately 10,15, and 30 days for tumors to reach a volume of $80-100 \mathrm{~mm}^{3}$ in the 1E6, 1E5, and 1E4 models, respectively, while it took approximately 80 days for each tumor on the autochthonous model to reach $100 \mathrm{~mm}^{3}$ (Fig. 1c).

To test if tumor latency influences immune cell infiltration or phenotype, immunophenotyping was performed on tumors generated in each breast cancer model. Tumors were harvested when they reached $100 \mathrm{~mm}^{3}$ for baseline immune profiling because this is generally the size of tumors when animals are enrolled into efficacy studies. In addition, tumors that are $100 \mathrm{~mm}^{3}$ allow for immune cell analysis without areas of tumor necrosis. Flow cytometry revealed significant difference in the frequency of leukocytes (CD45 ${ }^{+}$cells) within the tumor as a percentage of total viable cells (Fig. 1d). Tumors with the shortest latency (1E6 and 1E5 models) had the highest absolute number of infiltrating leukocytes as measured by $\mathrm{CD}_{4} 5^{+}$cells out of total viable cells (Fig. 1e). Strikingly, in the 1E6 and 1E5 models, immune cells were the major cell type within tumors, representing more than $60 \%$ of the total cells in the tumor which may represent an acute inflammatory response from the inoculation (Fig. 1e). T-cells (CD3 ${ }^{+}$) and myeloid cells $\left(\mathrm{CD} 11 \mathrm{~b}^{+}\right)$made up the majority of the $\mathrm{CD} 45^{+}$cells; with less than $5 \%$ contribution from other $\mathrm{CD} 45^{+}$cells (Fig. 1f-h). Myeloid cells (percent of $\mathrm{CD} 11 \mathrm{~b}^{+}$cells out of total of viable cells) were consistent in their frequency between models, however the autochthonous model had significantly fewer myeloid cells than the 1E6 and 1E5 models (Fig. 1d,g). T-cells (percent of $\mathrm{CD}^{+}{ }^{+}$cells out of total of viable cells) accounted for a substantial fraction of the $\mathrm{CD} 45^{+}$cells in the 1E6 and 1E5 syngeneic models and was less robust in the 1E4 and transgenic models (Fig. 1d,h). This resulted in differences in the ratio of Tcells to myeloid cells, where tumor models with a shorter tumor latency had the highest ratios. The 1E6 model had 5.63 T-cells to every 1 myeloid cell whereas the autochthonous model had $2.01 \mathrm{~T}$-cells for every 1 myeloid cell (Fig. 1i). Additionally, a correlation was observed between tumor latency and the number of T-cells within the tumor (Fig. 1j). These experiments were repeated with a newly generated batch of inoculum and both inoculum 1 and inoculum 2 yielded similar results (Supl. Fig. 1c).

\section{Subset characterization reveals differences in tumor T-cell immune populations among the 4 distinct breast cancer models.}

To confirm the absolute numbers of immune cells in tumors we determined the proportion of T-cells per gram of tumor tissue as well as their frequency out of $\mathrm{CD} 45^{+}$cells. The extent of T-cell infiltration out of gram of tissue (Fig. 2a) was similar to the findings out of total viable cells (Fig. $1 \mathrm{~h}$ ), which reflects the absolute increase in numbers of T-cells out of all cells in the tumor. This trend was consistent with the absolute numbers of $\mathrm{CD}^{+}$and $\mathrm{CD} 4^{+}$T-cells (Fig. 2a). The difference in absolute numbers of T-cells prompted further investigation into immune cell subpopulations and their respective phenotypes. Using flow cytometry, T-cell populations were characterized out of $\mathrm{CD}^{+}$cells including $\mathrm{CD}^{+}{ }^{+} \mathrm{FoxP} 3^{+}(\mathrm{T}-$ 
regulatory cells; Tregs), $\mathrm{CD}^{+}{ }^{+} \mathrm{FoxP} 3^{-}$and $\mathrm{CD} 8^{+} \mathrm{T}$-cells (gating strategy shown in Supl. Fig. 2a). The absolute number of CD8+ and CD4+ cells in the syngeneic models was significantly higher than the autochthonous model (Fig 2a). The same pattern emerged when gated as a frequency out of $C D 45^{+}$cells (Fig. 2b). Tregs are responsible for dampening the cytotoxic $T$ lymphocyte (CTL) responses and are characterized by expression of the transcription factor FoxP3. ${ }^{61}$ The Treg population increased in the 1E5 and 1E4 models (Fig. 3a,b) and there was no change in the frequency of CD8 T-cells out of total CD3 ${ }^{+}$ cells (Fig. 3c-e). The ratio of $\mathrm{CD} 4^{+} \mathrm{CD} 3^{+}$cells over $\mathrm{CD} 8^{+} \mathrm{CD} 3^{+}$cells was highest in the $1 \mathrm{E} 6$ model (Fig. $3 f$ ). A different gating strategy was used to identify the frequency of FoxP3 ${ }^{+}$cells out of $\mathrm{CD}^{+}{ }^{+} \mathrm{T}$-cells (Tregs) as well as the frequency of granzyme $b(\mathrm{GrB})^{+} \mathrm{CD} 8^{+} \mathrm{T}$-cells (cytotoxic $T$ lymphocytes; $\mathrm{CTLs}$ ). This revealed that the $1 \mathrm{E} 5$ and 1E4 models again had the highest frequency of Tregs whereas the autochthonous model had the highest frequency of $\mathrm{GrB}^{+} \mathrm{CD} 8^{+} \mathrm{CTLs}$ (Fig. 3g,h). This resulted in a significant increase in the CTL:Treg ratio in the autochthonous model compared to the 1E5 and 1E4 model (Fig. 3i).

\section{Subset characterization reveals differences in tumor myeloid cell populations among the breast cancer models.}

Further analysis of myeloid $\left(\mathrm{CD} 11 \mathrm{~b}^{+}\right)$cells revealed significant differences of myeloid subpopulations between the four distinct tumor models (Fig. 4a-e). While the autochthonous model had the fewest number of myeloid cells out of viable cells (Fig. 1g), as well as out of gram of tumor (Fig. 2c), it had the highest frequency of myeloid cells $\left(\mathrm{CD} 11 \mathrm{~b}^{+}\right)$and macrophages $\left(\mathrm{F} 4 / 80^{+}\right)$out of total $\mathrm{CD} 45^{+}$immune cells (Fig. 2d). The other myeloid cell populations $\left(\mathrm{Gr}^{+} \mathrm{CD} 11 \mathrm{~b}^{+}\right.$and $\left.\mathrm{CD} 11 \mathrm{c}^{+}\right)$were relatively low and unchanged between the distinct models, except for a small, yet significant increase in MDSCs $\left(\mathrm{Gr} 1^{+} \mathrm{CD} 11 \mathrm{~b}^{+}\right)$in the $1 \mathrm{E} 6$ compared to the $1 \mathrm{E} 4$ model (Fig. $\left.2 \mathrm{~d}\right)$. There was a 1:1 ratio of $\mathrm{CD} 11 \mathrm{~b}^{+}$to $\mathrm{CD}^{+}$ of $\mathrm{CD} 45^{+}$cells in the transgenic model, which was the highest ratio among the four models (Fig. 2e). Therefore, while there are fewer immune cells in the TME of MMTV-PyMT tumors compared to the syngeneic tumors, the percent of myeloid cells out of CD45 cells is highest. The autochthonous and 1E4 models had the highest proportion of $\mathrm{Gr}^{+}$cells out of $\mathrm{CD} 11 \mathrm{~b}^{+}$cells (Fig. 4a); this phenotype is generally characterized as myeloid derived suppressor cells. ${ }^{62,63}$ The $\mathrm{F} 480^{+}$cells in the autochthonous model were largely PD-L1 negative, in contrast to $\mathrm{F}_{480^{+}}$cells in the syngeneic models, which had the highest proportion of PD-L1 positive F4/80 cells (Fig. 4b,c,e). A different gating strategy was used to assess PD$\mathrm{L} 1^{+}$cells out of $\mathrm{CD} 11 \mathrm{~b}^{+}$cells as well as of $\mathrm{CD} 45^{-}$cells and demonstrated that the frequency of PD-L1 expression on myeloid cells was lowest in the autochthonous model, and there was no change on CD45 negative cells (Fig. 4f,g). Interestingly, the autochthonous model had the highest frequency of PD-1+ ${ }^{+}$ cells out of $\mathrm{CD}^{+} \mathrm{CD} 45^{+}$cells (Fig. $4 \mathrm{~h}$ ).

Our group previously described that using macrophage targeting compounds to convert pro-tumor macrophages to an anti-tumor phenotype induced reduction of primary and metastatic tumors in the MMTV-PyMT autochthonous model, indicating that the myeloid cell population is a major contributor to 
disease progression. ${ }^{43,64}$ The phenotype of tumor macrophages has previously been shown to correspond to drug sensitivity and disease outcome in this model, therefore we further investigated macrophage phenotype across the different models. ${ }^{43,44}$ Markers CD40, CD80 and CD86 were used to identify "M1"-like macrophages and CD206 was used as a marker of "M2"-like macrophages. The autochthonous model and the 1E4 model had the highest frequency of M1-like myeloid and macrophage populations (Fig. 4i and Supl. Fig. 3a-c) suggesting that the 1E6 and 1E5 models had more suppressive myeloid cells, in line with their higher frequencies of PD-L1+ myeloid cells (Fig. 4f).

\section{The breast cancer models have distinct tumor immune transcriptional profiles.}

To interrogate mRNA transcripts expressed by cells in each of the distinct tumors, transcriptional profiling was performed by NanoString analysis (Supplemental Fig. 4). Since the analysis was performed on bulk tumor tissue, the data would correspond most similarly to flow cytometry analysis out of total viable cells and per gram of tumor tissue. The autochthonous tumors clustered most closely with the 1E4 tumors, while and the 1E6 and 1E5 tumors clustered together (Supplemental Fig. 4c,d Supplemental Fig. 8b, d). Cell type score analysis validated the flow cytometry data, where the 1E6 model had the highest scores for all immune cell types profiled, including T-cells and macrophages (Fig. 5a-c and Supplemental Fig. 4e). In addition, there was no change in neutrophils but the 1E6 and 1E5 model had the highest scores for dendritic cells, and the 1E6 model had the highest score for activated natural killer cells (NK CD56dim; Supplemental Fig. 4f).

Unsupervised hierarchical clustering showed 1E6 and 1E5 models clustered most similarly and the 1E4 and autochthonous models clustered most similarly on activated pathway scores (Fig. 5c). Pathway analysis further revealed increased scores for immune related pathways including both adaptive and innate immunity, as well as macrophage and T-cell function. In general, the $1 \mathrm{E} 6$ and $1 \mathrm{E} 5$ models had the highest scores for all immune related pathways (Fig. 5d and Supplemental Fig. 5, Table 1-2), likely due to the increased number of immune cells in the tumors that were identified by flow cytometry. The most striking differences were observed when comparing the autochthonous model with the 1E6 model, where multiple transcripts associated with antigen presentation and immune signaling were increased in the $1 \mathrm{E6}$ model (Supplemental Fig. 6a-f). More specifically, the 1E6 model had the highest levels of Cd274 (PD-L1), Ctla4, Ccr2 and Ccl2 (Fig. 5e).

\section{The distinct models of MMTV-PyMT breast cancer respond differently to immune checkpoint blockade.}

We and others have previously shown that the MMTV-PyMT murine model of breast cancer is resistant to checkpoint blockade as a monotherapy. ${ }^{43,65}$ Here, the MMTV-PyMT model had the least number of infiltrating leukocytes and lymphocytes within the TME compared to the 3 syngeneic models. Since the syngeneic models had a higher absolute number of T-cells, as well as a higher proportion of T-cells expressing PD-1 and myeloid cells that express PD-L1, we hypothesized that the syngeneic models would demonstrate enhanced responses to ICB. Indeed, the $1 \mathrm{E} 6$ model, which had the highest frequency of PD$\mathrm{L} 1^{+}$myeloid cells, was the only model among the four that responded to anti-PD-L1 and anti-CTLA-4 as 
monotherapy after 14 days of treatment as seen by delayed tumor progression (Fig. 6, Supplemental Fig. 7). The combination of anti-PD-L1 plus anti-CTLA-4 was significant over vehicle control but not over either monotherapy. This resulted in a small yet significant increase in overall survival (Fig. 6a). The 1E5 model had a small yet significant decrease in total tumor burden with the anti-CTLA-4 monotherapy at day 14 , but did not translate to an increase in overall survival; however the combination of anti-CTLA-4 plus anti-PD-L1 induced a reduction of tumor burden at 14 days that led to improved overall survival (Fig. $6 \mathrm{~b}$, Supplemental Fig. 7b). Both the 1E4 and the autochthonous model were resistant to monotherapy as well as combination therapy (Fig. 6c-d, Supl. Fig. 7c-d).

To test if cell density is the largest driver of response to ICB or if residual immune cells in the inoculum account for these differences, $\mathrm{CD} 45^{+}$cells were successfully removed from the inoculum (referred to as sorted) and compared to the original inoculum for their growth rate and response to ICB (Fig. 7a-b). The unsorted and sorted inoculum grew at similar rates in all three models (1E6, 1E5, and 1E4) when injected into immunocompetent FVB/NJ mice (Fig. 7c). In nude mice, which lack a functional immune system, the unsorted cells grew faster in nude mice compared to FVB/NJ mice, as expected (Fig. 7c). The nude mice reached endpoint in a cell density-dependent manner (Fig. 7d). Next, unsorted (original) and sorted inoculum were injected into immunocompetent mice at either 1E6 or 1E4 and when tumors reached 100 $\mathrm{mm}^{3}$ they were treated with anti-CTLA4 plus anti-PD-L1. The unsorted inoculum responded as we previously demonstrated (Fig. 6a) however the sorted inoculum in the 1E6 model failed to respond to ICB (Fig. 7e). This suggests that the immune cells co-injected with tumor cells may play a key role in activating an acute immune response that sensitizes the tumor to ICB.

To gain insight into what may correlate with ICB response, tumors were regrouped into either predicted responders (1E6 and 1E5 tumors) or predicted non-responders (1E4 and autochthonous; Supl. Fig. 8a). Predicted responders and non-responders clustered by immune cell score, where predicted responders had higher scores related to CD45, T-cells, cytotoxic T-cells, NK CD56 dim cells, macrophages and dendritic cells (DCs) (Supl. Fig. 8b,c). Responders and non-responders also clustered in the pathway analysis, where the inflammation pathway revealed significant differences between the responders and non-responders (Supl. Fig. 8d,e). This data suggests that total immune cell infiltrate as well exhaustive immune cell phenotypes may correspond to the success of ICB therapy within the context of these breast cancer models (Supl. Fig. 9).

\section{Discussion}

Mouse models are critical to the rapid and successful translation of preclinical findings to the clinic yet are currently lacking. In addition, there is a critical need for biomarkers to predict response to ICB in breast cancer. Given the substantial heterogeneity of the TME, conclusions based on specific mouse models might limit generalizations, especially regarding the detailed characterization of molecular signaling mechanisms. The MMTV-PyMT autochthonous model has been extensively characterized and is one of the few models available to study Luminal B breast cancer. MMTV-PyMT mice develop spontaneous mammary tumors that closely resemble the progression and morphology of human breast 
cancers. ${ }^{41,52,53}$ Notably, gene expression profiling has revealed that MMTV-PyMT tumors cluster closely with ERa-negative 'luminal' human breast cancers ${ }^{66}$, which is a gene signature similar to the luminal-AR (LAR) TNBC subtype characterized by high AR expression ${ }^{67}$ and the molecular apocrine ER/PR negative, but AR+ tumors described prior to molecular subtyping. ${ }^{68}$ The pathology of the autochthonous murine breast tumors provides numerous ways to model human breast cancer in vivo. Here we report that the $1 \mathrm{E} 6$ and $1 \mathrm{E} 5$ corresponding syngeneic models do not recapitulate the autochthonous model. Our findings presented here are essential for future preclinical studies and translation to the clinic.

Here we exploited the MMTV-PyMT syngeneic mouse model to make two major findings. First, the initiating conditions of the tumor (in this case, the number of cells in the inoculum) can dramatically alter the tumor immune microenvironment. Second, we found that these differences in the TME were closely related to the quality of ICB responses (Supl. Fig. 9). We used cells derived from tumors that spontaneously arise in the MMTV-PyMT murine model of breast cancer to generate three distinct syngeneic models using 1E6, 1E5 or 1E4 cells injected into the mammary fat pad of wild-type FVB/NJ mice. Our findings are the first to report a detailed characterization of the difference in the TME as a variable of the number of cells injected to generate syngeneic tumors. Importantly, we find that while the $1 \mathrm{E} 6$ and $1 \mathrm{E} 5$ models responded to ICB, the 1E4 and MMTV-PyMT models are resistant. The ICB-sensitive tumors demonstrated that protection from the inhibitory effects of Tregs and the presence of high numbers of T-cells and macrophages paired with enhanced antigen processing capabilities correlated with response to ICB. These data support our hypothesis that in addition to T-cells, macrophages and other myeloid cells are required to play a critical role in initiating an anti-tumor immune response. In the clinic, tumors with these characteristics may have greater therapeutic responses to ICB.

The 1E6 and 1E5 tumors had the highest absolute number of T-cells. T-cells have been used as a prognostic biomarker, yet in this case, infiltration of T-cells is likely a response to an acute inflammatory response and not related to T-cell recruitment in human tumors. Regardless, the response to ICB correlated with increased T-cells (Fig. 3a). Interestingly, there was no correlation between ICB-sensitivity and frequency of CTL numbers or proportions, as the autochthonous model had the highest frequency but was resistant to ICB. The functional activity of T-cells depends largely on the expression of costimulatory molecules, peptide-MHC complexes, MHC class I molecules, and expression of checkpoint markers (PD-1 and CTLA4). ${ }^{69}$ T-cells secrete cytokines to promote a differential effector function. Activated T-cells (Th1 type) can secrete IL-2, TNFa and IFNy, which in turn induce cytotoxic function of CD8+ T-cells and promote phagocytosis through co-stimulatory markers on macrophages and other antigen presenting cells (CD40, CD86, and CD80). ${ }^{70,71}$ In contrast, secretion of IL-4 -6 - $10-13$ by Th2 CD4 ${ }^{+}$ T-cells can promote T-cell anergy and inhibit the activation of CTLs. ${ }^{44}$ Tregs correlate with poor prognosis in a variety of epithelial tumor types possibly as a result of dampening T-cell immunity in response to cancer associated antigens. ${ }^{72,73}$ Here, Tregs did not appear to correlate with ICB responses. Another factor worth considering is the low numbers of myeloid cells to facilitate antigen presentation in the autochthonous model, compared to the 1E6 model, which may render the CTLs ineffective in mediating the response to ICB in the autochthonous model. The antigen presentation signature and corresponding 
genes were significantly increased in the ICB-sensitive models (1E6 and 1E5) compared to the ICBresistant models (1E4 and autochthonous). This may reflect the fact that the ICB-sensitive tumors received a higher density of cells and therefore a potentially higher antigen load was delivered to mice; or it may represent that more $\mathrm{CD} 45+$ cells (absolute number) are injected into mice with the inoculum at 1E6. When CD45 cells are removed from the inoculum prior to injection into wild-type FVB/N mice at a density of 1E6, the tumors are no longer sensitive to ICB (Fig. 7e), which may indicate that the residual immune cells injected into the mice in the inoculum activate an immune response and facilitated recruitment of host T-cells. Therefore, increased TILs and antigen presentation may be falsely increased in the sensitive models and may not represent naturally occurring tumors.

An important question, that is currently unknown is if the absolute number of myeloid cells within the TME or the proportion of myeloid cells of total CD45 immune cells is a more important factor for ICB efficacy. The data here suggest that the former is a stronger predictor of response, and that the phenotype might not be as critical since the ratio of M1:M2 macrophages was higher in the ICB-resistance models (albeit lower absolute numbers of myeloid cells). Further work to understand TAM phenotype should be carefully noted by their function, signaling pathways and expression of extracellular markers. We found that the $1 \mathrm{E} 6$ and $1 \mathrm{E} 5$ models had the highest absolute number of $\mathrm{CD} 11 \mathrm{~b}^{+}$myeloid and $\mathrm{F} 480^{+}$ macrophages, yet the differences were not as pronounced as the difference in T-cells. Macrophages play an essential role in T-cell activation by presenting antigen and providing activating and stimulatory cytokines essential for T-cell function. ${ }^{69}$ In addition, macrophages can mediate antibody dependent cellular toxicity of cancer cells ${ }^{74}$ as well as eliminate cancer cells through FcgR-mediated phagocytosis. ${ }^{75}$ However, TAMs can also dampen effector T-cell function by producing IL-10 that in turn increase their own PD-L1 expression and suppresses cytotoxic T-cell responses. ${ }^{76}$ The myeloid cells in the 1E6 and 1E5 tumors were more slightly more suppressive than those found in the 1E4 and autochthonous tumor models; indicated by a higher proportion of myeloid cells expressing PD-L1 ${ }^{+}$(Fig. 4f), as well as a lower ratio of M1:M2 macrophages that suggested more M2-like macrophages (Supl. Fig. 3). In line with these observations, we found that transcript levels related to $\mathrm{Ccl} 2$ and its receptor were higher in the ICBsensitive models. CCL2 is a cytokine largely known for its involvement in the recruitment of CCR2+ monocytes from the bone marrow to other sites in the body where they differentiate into macrophages. ${ }^{77,78}$ Additionally, CCL2 has been shown to recruit monocytes and macrophages to breast tumors and to facilitate breast cancer metastasis. ${ }^{79,80}$ The CCL2/CCR2 axis may represent a unique opportunity for anti-cancer therapy and work in this area is already being explored. ${ }^{81,82}$ The combination of CCL2 antagonism with anti-PD-1 has demonstrated efficacy in some mouse models. ${ }^{83}$ Taken together, the differences we found in the myeloid compartment was not as striking as those observed for T-cells, and importantly studies have revealed similar outcome for myeloid-targeting strategies between these syngeneic and autochthonous models, where they appear to be able to be used interchangeably. ${ }^{54,84}$ This suggests that myeloid based immunotherapy studies, but not T-cell ICB studies may be suitable in the $1 \mathrm{E} 6$ model, but was not directly tested here. 
Other studies have shown that the inoculated cell density of 4T1 cells is a determinant of the growth dynamics and metastatic potential of the cells, where injecting fewer cells resulted extending the time of tumor development to result in $100 \%$ metastasis to study metastatic tumors. ${ }^{85}$ Table 4 summarizes the use of syngeneic models of breast cancer (4T1, EMT6, and MMTV-PyMT) reported to evaluate PD-(L)1 and CTLA4 blockade efficacy. We observe a lack of standardization of the number of cells inoculated (ranging 5E4-5E6), as well as days after inoculation (7-24 days) and tumor size (40-400 $\mathrm{mm}^{3}$ ), reported at the start of treatment. In an effort to best represent the human disease, the use of autochthonous models and/or syngeneic models that most closely resemble the disease is critical. Our study has shown that the most common strategy of generating syngeneic mouse models by inoculating $1 \mathrm{E} 6 \mathrm{cells}$, in fact, does not resemble the tumor microenvironment of its autochthonous host. This may result in experimental outcomes that will not have a clinical translation.

We observed a correlation between baseline PD-L1 expression of myeloid cells (Fig. 4f) but not CD45 negative cells (Fig. $4 \mathrm{~g}$ ) and response to ICB. This is an important observation seeing as inclusion criteria for some ICB treatment and/or clinical trials require PD-L1 expression (NCT03258788, NCT02536794). NanoString gene expression analysis of the 1E6 tumors also revealed elevated levels of both CD274 (PDL1) and CTLA-4 (Fig. 5e); which corresponds with the response to anti-CTLA-4 and anti-PD-L1 monotherapy (Fig. 6a). A limitation to this work is that the TME was not assessed after ICB, which may reveal additional changes to the TME that correlate with response to therapy.

\section{Conclusion}

The evasion of immune surveillance is a challenge in breast ICB therapy that warrants further investigation. Mechanistic understanding of how the TME promotes tumor progression will be critical to understanding which cell populations play the most influential role in promoting an immune escape. However, comprehensive immunophenotyping and response to ICB of mouse models are currently lacking. Here we performed immunophenotyping of the MMTV-PyMT autochthonous model compared to 3 syngeneic models derived from MMT-PyMT tumors and tested their response to anti-CTLA4 and antiPD-L1 therapy. We've revealed that the TME of tumors from the 1E6 and 1E5 syngeneic models are vastly different from the 1E4 and autochthonous models. We've uncovered that innate immunity and antigen processing may play a vital role in determining response to checkpoint blockade yet may be an artificial response in the ICB-sensitive models $(1 \mathrm{E} 6,1 \mathrm{E} 5)$. Further work to characterize the signals within the TME that promote immune evasion will be vital to advancing checkpoint blockade therapy for the treatment of breast cancer. Shedding light on why the ICB-sensitive models are sensitive to ICB therapy and providing syngeneic models to study ICB resistance is a major advancement for the study of immunotherapy in breast cancer and represents a unique opportunity to further interrogate biomarkers of response to ICB.

\section{Supplemental Legends}

\section{Supplemental Figure 1. Immunophenotyping of cells used for generation of syngeneic murine models.}


Tumors from the MMTV-PyMT autochthonous model were used to generate inoculum to inject into wild type mice to generate 1E6, 1E5 and 1E4 syngeneic tumor models. Flow cytometry was performed on three separate batches of inoculum, which were used for each unique experiment and are shown as red (inoculum1), blue (inoculum2), and green (inoculum3). a. Gating strategy for flow cytometry b. Immune cell composition as a percent out of viable cells. c. Frequency of immune cell populations out of $C D 45^{+}$ cells. Graphs show mean \pm SEM. ${ }^{\star} P<0.05, * \star P<0.01, * \star \star P<0.001, * \star \star \star * 0.001$.

\section{Supplemental Figure 2. Example of FACs gating strategy.}

a. Gating strategy for flow cytometry.

\section{Supplemental Figure 3. Ratio of anti-tumor to pro-tumor macrophages.}

Tumors from the autochthonous MMTV-PyMT model were harvested and single-cell suspensions were generated. Cells (1E6, 1E5 or 1E4) were injected into mammary fat pad number 4 of FVB/NJ wild type mice. When the tumors reached $100 \mathrm{~mm}^{3}$, tumors were obtained and processed into single-cell suspension for immunophenotyping by flow cytometry to identify the ratio of classically activated macrophages $\mathrm{CD}_{40}{ }^{+}(\mathrm{a}), \mathrm{CD} 80^{+}(\mathrm{b})$, and $\mathrm{CD} 86^{+}(\mathrm{c})$ of $\mathrm{F} 4 / 80^{+}$to alternatively activated macrophages indicated by the mannose receptor, $\mathrm{CD} 206^{+}$of $\mathrm{F} 4 / 80^{+}$cells. Each data point represents an individual

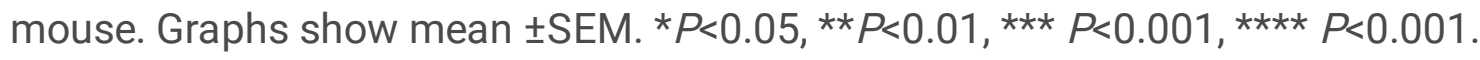

\section{Supplemental Figure 4. QC of mRNA expression analysis.}

Tumors from the autochthonous MMTV-PyMT model were harvested and single-cell suspensions were generated. Cells (1E6, 1E5 or 1E4) were injected into mammary fat pad number 4 of FVB/NJ wild type mice. When the tumors reached $100 \mathrm{~mm}^{3}$, RNA was extracted from tumors and NanoString analysis was performed. Data was analyzed using the Advanced Analysis 2.0 Module of the nSolver ${ }^{\mathrm{TM}}$ software (NanoString Technologies). The geNorm algorithm was used for selecting housekeeping genes and samples were normalized against positive controls. a. Normalization results shows consistency among selected housekeeping genes according to geNorm. b. Mean gene expression plotted versus variance. Housekeeping genes used in normalization are highlighted. c. Principle component analysis scored across the four models. The first two principal components explain $36 \%$ and $14 \%$ of variance respectively. d. Unsupervised hierarchical clustering was performed on all normalized transcripts above background. Orange cells indicate higher than average expression; blue cells indicate lower than average expression. e. A summary of cell types scores are shown across the four groups. e Quantification of cell types scores for neutrophils, dendritic cells, natural killer cells, and natural killer CD56dim are shown. Each data point represents an individual mouse. Graphs show mean \pm SEM. Unpaired two tailed $t$-test. ${ }^{\star} P<0.05, * \star P<0.01$, $\star \star \star * 00.001, \star \star \star \star ~ P<0.001$.

\section{Supplemental Figure 5. Quantification of pathway analysis by NanoString.}


Signature scores from the NanoString pathways analysis are shown. Graphs show mean \pm SEM with 4-5 mice per group. Unpaired two tailed t-test. ${ }^{*} P<0.05, \star \star P<0.01, \star \star \star ~ P<0.001, \star \star \star \star * P<0.001$.

\section{Supplemental Figure 6. Pathway analysis by NanoString.}

a. The heatmap summarizes the mRNA transcripts categorized by their respective pathway. Shown are the signature scoring differences of the syngeneic models compared to the transgenic model. Orange represents higher than average scores; blue represents lower than average scores. b-d. Volcano plots representing differential expression analysis of genes expressed in the 1E6 (top), 1E5 (middle), 1E4 model (bottom) compared to the transgenic model. Volcano plots show fold change (log2) versus $\log _{10} \mathrm{p}$ values. False discovery rate thresholds are shown ( $F D R<0.01, F D R<0.05, F D R<0.1$, and $F D R<0.5)$. e. Normalized log2 counts of transcripts related to the Antigen presentation pathway from the Nanostring pathways analysis are shown. f. Normalized log2 counts of transcripts related to the immune signaling pathway are shown. Graphs show mean \pm SEM with 4-5 mice per group. Unpaired two tailed $t$-test. $\star P<0.05, * \star P<0.01, \star \star \star x<0.001, \star \star \star \star * P<0.001$.

\section{Supplemental Figure 7. Syngeneic tumor treated with CTLA-4 and PD-L1 show significant differences in tumor fold change.}

Mice of similar tumor burden from each syngeneic model and the autochthonous model were randomized into four treatment groups: IgG2B, PD-L1 inhibitor, CTLA-4 inhibitor, and the combination of PD-L1 and CTLA-4 inhibitors. Tumors were measured every 2-3 days. Tumor volumes were measured and plotted as mean total tumor burden \pm SEM and tumor burden fold change were calculated at day 14 for

the 1E6 (a), 1E5 (b), 1E4 (c) and autochthonous (d) models. MMTV-PyMT autochthonous mice were enrolled into as study at about 80 days old and when each tumor reached $80-100 \mathrm{~mm}^{3}$. Mice have 10 mammary fat pads and tumor arise in all 10 fat pads. Tumors from mammary fat pad numbers 5 and 10 were excluded from our analysis. The sum of the volumes for tumors from the MMTV-PyMT autochthonous fat pads (1-4 and 5-9) were used and indicated as "total tumor burden". The syngeneic mice that had tumors that measured $80-100 \mathrm{~mm}^{3}$ were enrolled into an experiment. Shown are a summary of two individual experiments $n=3-7 /$ treatment groups. Two-way ANOVA multiple comparisons was performed $* P<0.05, * \star P<0.01, * \star \star * P<0.001, * \star \star \star * 0.001$.

\section{Supplemental Figure 8. Comparison of predicted responders vs. non-responders by NanoString analysis.}

The NanoString data was reanalyzed grouping mice into predicted responders (1E6 and 1E5) and predicted non-responders (1E4 and autochthonous). a. Principle component analysis. b. Heatmap of immune cell scores. c. Cell types scores are shown across the two groups. Each data point represents an individual mouse. d. Heatmap of pathway analysis showing predicted responders (gray) and nonresponders (orange). e. Differential expression of responders vs non-responders with the Inflammation Score Probe Set shown in red. Graphs show mean \pm SEM. Unpaired two tailed $t$-test. ${ }^{\star} P<0.05,{ }^{*} P<0.01$, $* \star \star$

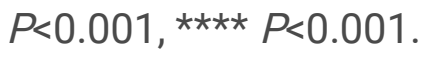




\section{Supplemental Figure 9. Summary slide of cellular immune components that correlate with response to immunotherapy.}

a. Key characteristics of immune cell subsets are summarized in the table and grouped by T-cells, myeloid cells, where " + " indicates significant changes, and blue and red represent significant increase and decrease expression, respectively, compared to the MMTV-PyMT model. Response to immune checkpoint blockade is also shown where blue and red represent significant increase and decrease response, respectively, compared to the vehicle control. b. Schematic representing the 4 tumor models and their immune composition. Figure created using biorender.com.

\section{Declarations}

\section{Ethical Approval and Consent to participate}

All mice were maintained within the Dana-Farer Cancer Center (DFCl) and all experiments were conducted under The Institutional Animal Care and Use Committee (IACUC).

\section{Consent for publication}

$\mathrm{n} / \mathrm{a}$

\section{Availability of supporting data}

The datasets used and/or analyzed during the current study are available from the corresponding author on reasonable request.

\section{Competing interests}

J.L.G. is a consultant for Glaxo-Smith Kline (GSK), Array BioPharma, Codagenix and Verseau, and receives sponsored research support from GSK, Eli Lilly and Array BioPharma. E.A.M has served on SABs for Astra-Zeneca/Medimmune, Celgene, Genentech, Genomic Health, Merck, Peregrine Pharmaceuticals, SELLAS Lifescience, and Tapimmune and has clinical trial support to her former institution (MDACC) from astra-zeneca/Medimmune, EMD-Serono, Galena Biopharma and Genentech as well as Genentech support to a SU2C grant, as well as sponsored Research Support to the laboratory from GSK and Eli Lilly. A.L. is a consultant for Astra-Zeneca, receives sponsored research support from Novartis, and serves on the scientific advisory board for Flash Therapeutics, Zentalis, and Dialectic. E.M. is an employee and stockholder of NanoString Technologies.

\section{Funding}

J.L.G is supported by a Career Enhancement Award through the National Institutes of Health $(\mathrm{NIH})$ grant Dana-Farber/Harvard Cancer Center Breast Cancer SPORE 5P50CA168504 and a Komen Career Catalyst Award CCR18547597. A.L, E.A.M and J.L.G are supported through the Ludwig Center at Harvard. 


\section{Authors contributions}

JCL, AKM, MGT, MO, AS, EC, EM and JLG performed all experiments and analyzed and interpreted the data. JCL, AKM and JLG wrote the manuscript. EAM, AL and JLG provided senior leadership. All authors read, edited and approved the final manuscript.

\section{Acknowledgements}

The authors thank Suzan Lazo for her technical help setting up flow cytometry panels. The authors are grateful for the expertise and assistance from the Dana-Farber Animal Research Facility and Flow Cytometry Core.

\section{References}

1 Waks, A. G. \& Winer, E. P. Breast Cancer Treatment: A Review. Jama 321, 288-300, doi:10.1001/jama.2018.19323 (2019).

2 Early Breast Cancer Trialists' Collaborative, G., Davies, C., Godwin, J., Gray, R., Clarke, M., Cutter, D. et al. Relevance of breast cancer hormone receptors and other factors to the efficacy of adjuvant

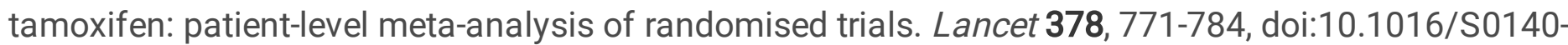
6736(11)60993-8 (2011).

3 Slamon, D. J., Leyland-Jones, B., Shak, S., Fuchs, H., Paton, V., Bajamonde, A. et al. Use of Chemotherapy plus a Monoclonal Antibody against HER2 for Metastatic Breast Cancer That Overexpresses HER2. New England Journal of Medicine 344, 783-792, doi:10.1056/nejm200103153441101 (2001).

4 Correa, C., Harris, E. E., Leonardi, M. C., Smith, B. D., Taghian, A. G., Thompson, A. M. et al. Accelerated Partial Breast Irradiation: Executive summary for the update of an ASTRO Evidence-Based Consensus Statement. Pract Radiat Oncol 7, 73-79, doi:10.1016/j.prro.2016.09.007 (2017).

5 O'Shaughnessy, J. Extending survival with chemotherapy in metastatic breast cancer. Oncologist 10 Suppl 3, 20-29, doi:10.1634/theoncologist.10-90003-20 (2005).

6 Emens, L. A. Breast Cancer Immunotherapy: Facts and Hopes. Clin Cancer Res 24, 511-520, doi:10.1158/1078-0432.CCR-16-3001 (2018).

7 Das, R., Verma, R., Sznol, M., Boddupalli, C. S., Gettinger, S. N., Kluger, H. et al. Combination therapy with anti-CTLA-4 and anti-PD-1 leads to distinct immunologic changes in vivo. J Immuno/ 194, 950-959, doi:10.4049/jimmunol.1401686 (2015).

8 Pardoll, D. M. The blockade of immune checkpoints in cancer immunotherapy. Nat Rev Cancer 12, 252-264, doi:10.1038/nrc3239 (2012). 
9 Hodi, F. S., O'Day, S. J., McDermott, D. F., Weber, R. W., Sosman, J. A., Haanen, J. B. et al. Improved survival with ipilimumab in patients with metastatic melanoma. N Engl J Med 363, 711-723, doi:10.1056/NEJMoa1003466 (2010).

10 Schadendorf, D., Hodi, F. S., Robert, C., Weber, J. S., Margolin, K., Hamid, O. et al. Pooled Analysis of Long-Term Survival Data From Phase II and Phase III Trials of Ipilimumab in Unresectable or Metastatic Melanoma. J Clin Onco/33, 1889-1894, doi:10.1200/JC0.2014.56.2736 (2015).

11 Tsao, H., Atkins, M. B. \& Sober, A. J. Management of cutaneous melanoma. N Engl J Med 351, 998-1012, doi:10.1056/NEJMra041245 (2004).

12 Lipson, E. J. \& Drake, C. G. Ipilimumab: an anti-CTLA-4 antibody for metastatic melanoma. Clin Cancer Res 17, 6958-6962, doi:10.1158/1078-0432.CCR-11-1595 (2011).

13 Brahmer, J. R., Tykodi, S. S., Chow, L. Q., Hwu, W. J., Topalian, S. L., Hwu, P. et al. Safety and activity of anti-PD-L1 antibody in patients with advanced cancer. N Engl J Med 366, 2455-2465, doi:10.1056/NEJMoa1200694 (2012).

14 Powles, T., Foreshew, S. J., Shamash, J., Sarwar, N., Crabb, S., Sahdev, A. et al. A phase lb study investigating the combination of everolimus and dovitinib in vascular endothelial growth factor refractory clear cell renal cancer. Eur J Cancer 50, 2057-2064, doi:10.1016/j.ejca.2014.04.021 (2014).

15 Baumeister, S. H., Freeman, G. J., Dranoff, G. \& Sharpe, A. H. Coinhibitory Pathways in Immunotherapy for Cancer. Annu Rev Immuno/ 34, 539-573, doi:10.1146/annurev-immunol-032414112049 (2016).

16 Postow, M. A., Callahan, M. K. \& Wolchok, J. D. Immune Checkpoint Blockade in Cancer Therapy. J Clin Onco/ 33, 1974-1982, doi:10.1200/JC0.2014.59.4358 (2015).

17 Topalian, S. L., Drake, C. G. \& Pardoll, D. M. Immune checkpoint blockade: a common denominator approach to cancer therapy. Cancer Cel/ 27, 450-461, doi:10.1016/j.ccell.2015.03.001 (2015).

18 Vokes, E. E., Ready, N., Felip, E., Horn, L., Burgio, M. A., Antonia, S. J. et al. Nivolumab versus docetaxel in previously treated advanced non-small-cell lung cancer (CheckMate 017 and CheckMate 057): 3-year update and outcomes in patients with liver metastases. Ann Oncol 29, 959-965, doi:10.1093/annonc/mdy041 (2018).

19 Antonia, S. J., Villegas, A., Daniel, D., Vicente, D., Murakami, S., Hui, R. et al. Overall Survival with Durvalumab after Chemoradiotherapy in Stage III NSCLC. N Engl J Med 379, 2342-2350, doi:10.1056/NEJMoa1809697 (2018).

20 Overman, M. J., McDermott, R., Leach, J. L., Lonardi, S., Lenz, H. J., Morse, M. A. et al. Nivolumab in patients with metastatic DNA mismatch repair-deficient or microsatellite instability-high colorectal cancer 
(CheckMate 142): an open-label, multicentre, phase 2 study. Lancet Oncol 18, 1182-1191, doi:10.1016/S1470-2045(17)30422-9 (2017).

21 Davis, A. A. \& Patel, V. G. The role of PD-L1 expression as a predictive biomarker: an analysis of all US Food and Drug Administration (FDA) approvals of immune checkpoint inhibitors. Journal for ImmunoTherapy of Cancer 7, 278, doi:10.1186/s40425-019-0768-9 (2019).

22 Wolchok, J. D., Chiarion-Sileni, V., Gonzalez, R., Rutkowski, P., Grob, J. J., Cowey, C. L. et al. Overall Survival with Combined Nivolumab and Ipilimumab in Advanced Melanoma. N Engl J Med 377, 13451356, doi:10.1056/NEJMoa1709684 (2017).

23 Postow, M. A., Callahan, M. K. \& Wolchok, J. D. Immune Checkpoint Blockade in Cancer Therapy. Journal of clinical oncology : official journal of the American Society of Clinical Oncology 33, 1974-1982, doi:10.1200/JC0.2014.59.4358 (2015).

24 Larkin, J., Chiarion-Sileni, V., Gonzalez, R., Grob, J. J., Cowey, C. L., Lao, C. D. et al. Combined Nivolumab and Ipilimumab or Monotherapy in Untreated Melanoma. N Engl J Med 373, 23-34, doi:10.1056/NEJMoa1504030 (2015).

25 Cella, D., Grünwald, V., Escudier, B., Hammers, H. J., George, S., Nathan, P. et al. Patient-reported outcomes of patients with advanced renal cell carcinoma treated with nivolumab plus ipilimumab versus sunitinib (CheckMate 214): a randomised, phase 3 trial. Lancet Oncol 20, 297-310, doi:10.1016/s14702045(18)30778-2 (2019).

26 Antonia, S. J., López-Martin, J. A., Bendell, J., Ott, P. A., Taylor, M., Eder, J. P. et al. Nivolumab alone and nivolumab plus ipilimumab in recurrent small-cell lung cancer (CheckMate 032): a multicentre, openlabel, phase 1/2 trial. Lancet Oncol 17, 883-895, doi:10.1016/s1470-2045(16)30098-5 (2016).

27 Overman, M. J., Lonardi, S., Wong, K. Y. M., Lenz, H. J., Gelsomino, F., Aglietta, M. et al. Durable Clinical Benefit With Nivolumab Plus Ipilimumab in DNA Mismatch Repair-Deficient/Microsatellite Instability-High Metastatic Colorectal Cancer. J Clin Onco/36, 773-779, doi:10.1200/jco.2017.76.9901 (2018).

28 Colli, L. M., Machiela, M. J., Myers, T. A., Jessop, L., Yu, K. \& Chanock, S. J. Burden of Nonsynonymous Mutations among TCGA Cancers and Candidate Immune Checkpoint Inhibitor Responses. Cancer Res 76, 3767-3772, doi:10.1158/0008-5472.CAN-16-0170 (2016).

29 Bertucci, F., Ng, C. K. Y., Patsouris, A., Droin, N., Piscuoglio, S., Carbuccia, N. et al. Genomic characterization of metastatic breast cancers. Nature 569, 560-564, doi:10.1038/s41586-019-1056-Z (2019).

30 Cortés, J., Lipatov, O., Im, S. A., Gonçalves, A., Lee, K. S., Schmid, P. et al. KEYNOTE-119: Phase III study of pembrolizumab (pembro) versus single-agent chemotherapy (chemo) for metastatic triple 
negative breast cancer (mTNBC). Annals of Oncology 30, v859-v860, doi:10.1093/annonc/mdz394.010 (2019).

31 Adams, S., Loi, S., Toppmeyer, D., Cescon, D., De Laurentiis, M., Nanda, R. et al. Abstract PD6-10: KEYNOTE-086 cohort B: Pembrolizumab monotherapy for PD-L1-positive, previously untreated, metastatic triple-negative breast cancer (mTNBC). Cancer Research 78, PD6-10-PD16-10, doi:10.1158/1538-7445.sabcs17-pd6-10 (2018).

32 Emens, L. A., Cruz, C., Eder, J. P., Braiteh, F., Chung, C., Tolaney, S. M. et al. Long-term Clinical Outcomes and Biomarker Analyses of Atezolizumab Therapy for Patients With Metastatic Triple-Negative Breast Cancer: A Phase 1 Study. JAMA Onco/ 5, 74-82, doi:10.1001/jamaoncol.2018.4224 (2019).

33 Adams, S., Schmid, P., Rugo, H. S., Winer, E. P., Loirat, D., Awada, A. et al. Phase 2 study of pembrolizumab (pembro) monotherapy for previously treated metastatic triple-negative breast cancer (mTNBC): KEYNOTE-086 cohort A. Journal of clinical oncology 35, 1008-1008, doi:10.1200/JC0.2017.35.15_suppl.1008 (2017).

34 Schmid, P., Adams, S., Rugo, H. S., Schneeweiss, A., Barrios, C. H., Iwata, H. et al. Atezolizumab and Nab-Paclitaxel in Advanced Triple-Negative Breast Cancer. N Engl J Med 379, 2108-2121, doi:10.1056/NEJMoa1809615 (2018).

35 Emens, L., Loi, S., Rugo, H., Schneeweiss, A., Diéras, V., Iwata, H. et al. Abstract GS1-04: IMpassion130: Efficacy in immune biomarker subgroups from the global, randomized, double-blind, placebo-controlled, phase III study of atezolizumab $+<$ em $>$ nab $</$ em $>$-paclitaxel in patients with treatment-naïve, locally advanced or metastatic triple-negative breast cancer. Cancer Research 79, GS104-GS01-04, doi:10.1158/1538-7445.Sabcs18-gs1-04 (2019).

36 Cortes, J., Cescon, D. W., Rugo, H. S., Nowecki, Z., Im, S. A., Yusof, M. M. et al. Pembrolizumab plus chemotherapy versus placebo plus chemotherapy for previously untreated locally recurrent inoperable or metastatic triple-negative breast cancer (KEYNOTE-355): a randomised, placebo-controlled, double-blind, phase 3 clinical trial. Lancet 396, 1817-1828, doi:10.1016/S0140-6736(20)32531-9 (2020).

37 Rimm, D. L., Han, G., Taube, J. M., Yi, E. S., Bridge, J. A., Flieder, D. B. et al. Reanalysis of the NCCN PD-L1 companion diagnostic assay study for lung cancer in the context of PD-L1 expression findings in triple-negative breast cancer. Breast Cancer Res 21, 72, doi:10.1186/s13058-019-1156-6 (2019).

38 Luen, S. J., Salgado, R., Fox, S., Savas, P., Eng-Wong, J., Clark, E. et al. Tumour-infiltrating lymphocytes in advanced HER2-positive breast cancer treated with pertuzumab or placebo in addition to trastuzumab and docetaxel: a retrospective analysis of the CLEOPATRA study. Lancet Oncol 18, 52-62, doi:10.1016/S1470-2045(16)30631-3 (2017).

39 Gil Del Alcazar, C. R., Huh, S. J., Ekram, M. B., Trinh, A., Liu, L. L., Beca, F. et al. Immune Escape in Breast Cancer During In Situ to Invasive Carcinoma Transition. Cancer Discov 7, 1098-1115, 
doi:10.1158/2159-8290.Cd-17-0222 (2017).

40 Walunas, T. L., Lenschow, D. J., Bakker, C. Y., Linsley, P. S., Freeman, G. J., Green, J. M. et al. CTLA-4 can function as a negative regulator of T cell activation. Immunity 1, 405-413, doi:10.1016/10747613(94)90071-x (1994).

41 Lin, E. Y., Jones, J. G., Li, P., Zhu, L., Whitney, K. D., Muller, W. J. et al. Progression to malignancy in the polyoma middle $T$ oncoprotein mouse breast cancer model provides a reliable model for human diseases. Am J Pathol 163, 2113-2126, doi:10.1016/S0002-9440(10)63568-7 (2003).

42 Hollern, D. P. \& Andrechek, E. R. A genomic analysis of mouse models of breast cancer reveals molecular features of mouse models and relationships to human breast cancer. Breast Cancer Res 16, R59, doi:10.1186/bcr3672 (2014).

43 Guerriero, J. L., Sotayo, A., Ponichtera, H. E., Castrillon, J. A., Pourzia, A. L., Schad, S. et al. Class Ila HDAC inhibition reduces breast tumours and metastases through anti-tumour macrophages. Nature 543, 428-432, doi:10.1038/nature21409 (2017).

44 DeNardo, D. G., Barreto, J. B., Andreu, P., Vasquez, L., Tawfik, D., Kolhatkar, N. et al. CD4(+) T cells regulate pulmonary metastasis of mammary carcinomas by enhancing protumor properties of macrophages. Cancer Cell 16, 91-102, doi:10.1016/j.ccr.2009.06.018 (2009).

45 Qian, B. Z., Li, J., Zhang, H., Kitamura, T., Zhang, J., Campion, L. R. et al. CCL2 recruits inflammatory monocytes to facilitate breast-tumour metastasis. Nature 475, 222-225, doi:10.1038/nature10138 (2011).

46 Ruffell, B., Au, A., Rugo, H. S., Esserman, L. J., Hwang, E. S. \& Coussens, L. M. Leukocyte composition of human breast cancer. Proc Natl Acad Sci U S A 109, 2796-2801, doi:10.1073/pnas.1104303108 (2012).

47 Biswas, S. K. \& Mantovani, A. Macrophage plasticity and interaction with lymphocyte subsets: cancer as a paradigm. Nat Immuno/ 11, 889-896, doi:10.1038/ni.1937 (2010).

48 Solinas, G., Germano, G., Mantovani, A. \& Allavena, P. Tumor-associated macrophages (TAM) as major players of the cancer-related inflammation. J Leukoc Bio/ 86, 1065-1073, doi:10.1189/jlb.0609385 (2009).

49 Qian, B. Z. \& Pollard, J. W. Macrophage diversity enhances tumor progression and metastasis. Cell 141, 39-51, doi:10.1016/j.cell.2010.03.014 (2010).

50 Schmittnaegel, M., Rigamonti, N., Kadioglu, E., Cassara, A., Wyser Rmili, C., Kiialainen, A. et al. Dual angiopoietin-2 and VEGFA inhibition elicits antitumor immunity that is enhanced by PD-1 checkpoint blockade. Sci Trans/ Med 9, doi:10.1126/scitranslmed.aak9670 (2017). 
51 Messenheimer, D. J., Jensen, S. M., Afentoulis, M. E., Wegmann, K. W., Feng, Z., Friedman, D. J. et al. Timing of PD-1 Blockade Is Critical to Effective Combination Immunotherapy with Anti-OX40. Clin Cancer Res 23, 6165-6177, doi:10.1158/1078-0432.CCR-16-2677 (2017).

52 Fluck, M. M. \& Schaffhausen, B. S. Lessons in signaling and tumorigenesis from polyomavirus middle T antigen. Microbiol Mol Biol Rev 73, 542-563, Table of Contents, doi:10.1128/mmbr.00009-09 (2009).

53 Guy, C. T., Cardiff, R. D. \& Muller, W. J. Induction of mammary tumors by expression of polyomavirus middle T oncogene: a transgenic mouse model for metastatic disease. Mol Cell Biol 12, 954-961 (1992).

54 DeNardo, D. G., Brennan, D. J., Rexhepaj, E., Ruffell, B., Shiao, S. L., Madden, S. F. et al. Leukocyte complexity predicts breast cancer survival and functionally regulates response to chemotherapy. Cancer Discov 1, 54-67, doi:10.1158/2159-8274.CD-10-0028 (2011).

55 Ruffell, B., Chang-Strachan, D., Chan, V., Rosenbusch, A., Ho, C. M., Pryer, N. et al. Macrophage IL-10 blocks CD8+ T cell-dependent responses to chemotherapy by suppressing IL-12 expression in intratumoral dendritic cells. Cancer Cell 26, 623-637, doi:10.1016/j.ccell.2014.09.006 (2014).

56 de Mingo Pulido, Á., Gardner, A., Hiebler, S., Soliman, H., Rugo, H. S., Krummel, M. F. et al. TIM-3 Regulates CD103(+) Dendritic Cell Function and Response to Chemotherapy in Breast Cancer. Cancer Cell 33, 60-74.e66, doi:10.1016/j.ccell.2017.11.019 (2018).

57 Na, Y. R., Kwon, J. W., Kim, D. Y., Chung, H., Song, J., Jung, D. et al. Protein Kinase A Catalytic Subunit Is a Molecular Switch that Promotes the Pro-tumoral Function of Macrophages. Cell Rep 31, 107643, doi:10.1016/j.celrep.2020.107643 (2020).

58 Vandesompele, J., De Preter, K., Pattyn, F., Poppe, B., Van Roy, N., De Paepe, A. et al. Accurate normalization of real-time quantitative RT-PCR data by geometric averaging of multiple internal control genes. Genome Bio/ 3, RESEARCH0034, doi:10.1186/gb-2002-3-7-research0034 (2002).

59 Tomfohr, J., Lu, J. \& Kepler, T. B. Pathway level analysis of gene expression using singular value decomposition. BMC Bioinformatics 6, 225, doi:10.1186/1471-2105-6-225 (2005).

60 Danaher, P., Warren, S., Dennis, L., D'Amico, L., White, A., Disis, M. L. et al. Gene expression markers of Tumor Infiltrating Leukocytes. J Immunother Cancer 5, 18, doi:10.1186/s40425-017-0215-8 (2017).

61 Zou, W. Regulatory T cells, tumour immunity and immunotherapy. Nat Rev Immuno/ 6, 295-307, doi:10.1038/nri1806 (2006).

62 Damuzzo, V., Pinton, L., Desantis, G., Solito, S., Marigo, I., Bronte, V. et al. Complexity and challenges in defining myeloid-derived suppressor cells. Cytometry. Part B, Clinical cytometry 88, 77-91, doi:10.1002/cyto.b.21206 (2015). 
63 Veglia, F., Perego, M. \& Gabrilovich, D. Myeloid-derived suppressor cells coming of age. Nature Immunology 19, 108-119, doi:10.1038/s41590-017-0022-x (2018).

64 Mehta, A., Cheney, E.M., Hartl, C., Pantelidou, C., Oliwa, M., Castrillon, J.A., Lin, J-R., Hurst, K.E., Taveira, M., , Johnson, N.T., Oldham, W.M., Kalocsay, M., Berberich, M.J., Boswell, S.A., Kothari, A., Johnson, S., Dillon, D.A., Lipschitz, M., Rodig, S., Santagata, S., Garber, J.E., Tung, N., Thaxton, J.E., Mittendorf, E.A., Sorger, P.K., Shapiro, G.I., Guerriero, J.L. . Differentiating macrophages are regulated by PARP inhibitors and can be harnessed to overcome PARP-inhibitor resistance in BRCA-associated triplenegative breast cancer. Nature Cancer, doi:10.1038/s43018-020-00148-7 (In Press).

65 Allen, E., Jabouille, A., Rivera, L. B., Lodewijckx, I., Missiaen, R., Steri, V. et al. Combined antiangiogenic and anti-PD-L1 therapy stimulates tumor immunity through HEV formation. Sci Trans/ Med 9, eaak9679, doi:10.1126/scitranslmed.aak9679 (2017).

66 Shoushtari, A. N., Michalowska, A. M. \& Green, J. E. Comparing genetically engineered mouse mammary cancer models with human breast cancer by expression profiling. Breast Dis 28, 39-51, doi:10.3233/bd-2007-28105 (2007).

67 Lehmann, B. D., Bauer, J. A., Chen, X., Sanders, M. E., Chakravarthy, A. B., Shyr, Y. et al. Identification of human triple-negative breast cancer subtypes and preclinical models for selection of targeted therapies. J Clin Invest 121, 2750-2767, doi:10.1172/jci45014 (2011).

68 Doane, A. S., Danso, M., Lal, P., Donaton, M., Zhang, L., Hudis, C. et al. An estrogen receptornegative breast cancer subset characterized by a hormonally regulated transcriptional program and response to androgen. Oncogene 25, 3994-4008, doi:10.1038/sj.onc.1209415 (2006).

69 Guerriero, J. L. in International Review of Cell and Molecular Biology (Academic Press, 2018).

70 Stout, R. D. \& Bottomly, K. Antigen-specific activation of effector macrophages by IFN-gamma producing (TH1) T cell clones. Failure of IL-4-producing (TH2) T cell clones to activate effector function in macrophages. J Immunol 142, 760-765 (1989).

71 Espinosa-Cueto, P., Magallanes-Puebla, A., Castellanos, C. \& Mancilla, R. Dendritic cells that phagocytose apoptotic macrophages loaded with mycobacterial antigens activate CD8 T cells via crosspresentation. PLoS One 12, e0182126, doi:10.1371/journal.pone.0182126 (2017).

72 Curiel, T. J., Coukos, G., Zou, L., Alvarez, X., Cheng, P., Mottram, P. et al. Specific recruitment of regulatory $T$ cells in ovarian carcinoma fosters immune privilege and predicts reduced survival. Nat Med 10, 942-949, doi:10.1038/nm1093 (2004).

73 Kono, K., Kawaida, H., Takahashi, A., Sugai, H., Mimura, K., Miyagawa, N. et al. CD4(+)CD25high regulatory $\mathrm{T}$ cells increase with tumor stage in patients with gastric and esophageal cancers. Cancer Immunol Immunother 55, 1064-1071, doi:10.1007/s00262-005-0092-8 (2006). 
74 Simpson, T. R., Li, F., Montalvo-Ortiz, W., Sepulveda, M. A., Bergerhoff, K., Arce, F. et al. Fcdependent depletion of tumor-infiltrating regulatory $T$ cells co-defines the efficacy of anti-CTLA-4 therapy against melanoma. J Exp Med 210, 1695-1710, doi:10.1084/jem.20130579 (2013).

75 Gul, N., Babes, L., Siegmund, K., Korthouwer, R., Bogels, M., Braster, R. et al. Macrophages eliminate circulating tumor cells after monoclonal antibody therapy. J Clin Invest 124, 812-823, doi:10.1172/jci66776 (2014).

76 Kuang, D. M., Zhao, Q., Peng, C., Xu, J., Zhang, J. P., Wu, C. et al. Activated monocytes in peritumoral stroma of hepatocellular carcinoma foster immune privilege and disease progression through PD-L1. J Exp Med 206, 1327-1337, doi:10.1084/jem.20082173 (2009).

77 Fuentes, M. E., Durham, S. K., Swerdel, M. R., Lewin, A. C., Barton, D. S., Megill, J. R. et al. Controlled recruitment of monocytes and macrophages to specific organs through transgenic expression of monocyte chemoattractant protein-1. J Immunol 155, 5769-5776 (1995).

78 Kersten, K., Coffelt, S. B., Hoogstraat, M., Verstegen, N. J. M., Vrijland, K., Ciampricotti, M. et al. Mammary tumor-derived CCL2 enhances pro-metastatic systemic inflammation through upregulation of IL1 $\beta$ in tumor-associated macrophages. Oncoimmunology 6, e1334744-e1334744, doi:10.1080/2162402X.2017.1334744 (2017).

79 Kitamura, T., Qian, B.-Z., Soong, D., Cassetta, L., Noy, R., Sugano, G. et al. CCL2-induced chemokine cascade promotes breast cancer metastasis by enhancing retention of metastasis-associated macrophages. The Journal of experimental medicine 212, 1043-1059, doi:10.1084/jem.20141836 (2015).

80 Qian, B.-Z., Li, J., Zhang, H., Kitamura, T., Zhang, J., Campion, L. R. et al. CCL2 recruits inflammatory monocytes to facilitate breast-tumour metastasis. Nature 475, 222-225, doi:10.1038/nature10138 (2011).

81 Lim, S. Y., Yuzhalin, A. E., Gordon-Weeks, A. N. \& Muschel, R. J. Targeting the CCL2-CCR2 signaling axis in cancer metastasis. Oncotarget 7, 28697-28710, doi:10.18632/oncotarget.7376 (2016).

82 Cassetta, L. \& Pollard, J. W. Targeting macrophages: therapeutic approaches in cancer. Nat Rev Drug Discov 17, 887-904, doi:10.1038/nrd.2018.169 (2018).

83 Wang, Y., Zhang, X., Yang, L., Xue, J. \& Hu, G. Blockade of CCL2 enhances immunotherapeutic effect of anti-PD1 in lung cancer. Journal of bone oncology 11, 27-32, doi:10.1016/j.jbo.2018.01.002 (2018).

84 Strachan, D. C., Ruffell, B., Oei, Y., Bissell, M. J., Coussens, L. M., Pryer, N. et al. CSF1R inhibition delays cervical and mammary tumor growth in murine models by attenuating the turnover of tumorassociated macrophages and enhancing infiltration by CD8+ T cells. Oncoimmunology 2, e26968, doi:10.4161/onci.26968 (2013). 
85 Gregório, A. C., Fonseca, N. A., Moura, V., Lacerda, M., Figueiredo, P., Simões, S. et al. Inoculated Cell Density as a Determinant Factor of the Growth Dynamics and Metastatic Efficiency of a Breast Cancer Murine Model. PLoS One 11, e0165817, doi:10.1371/journal.pone.0165817 (2016).

\section{Tables}

Due to technical limitations, Tables 1-4 are only available as a download in the supplemental files section

\section{Figures}


a.

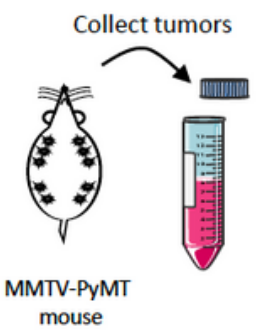

b.

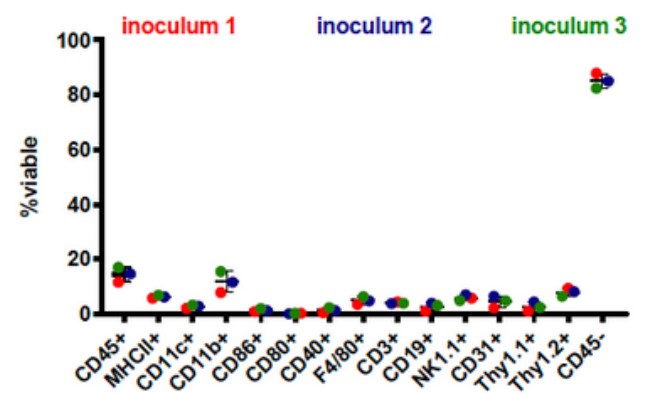

c.

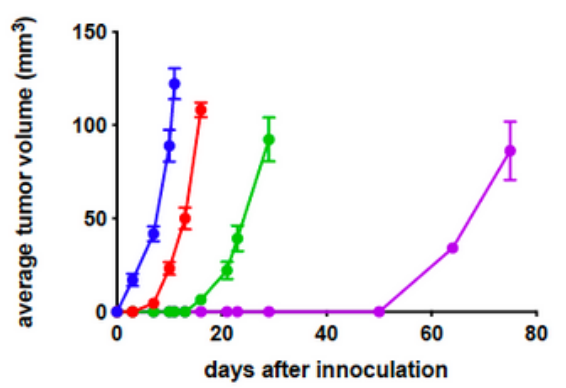

Immunophenotype by FACs:

1. Macrophage Panel

2. T-cell Panel

3. Checkpoint Marker Panel

Gene Expression: Nanostring

Enroll into efficacy study:

1. Vehicle

2. Anti-CTLA4

3. Anti-PD-L1

4. Anti-CTLA4 + Anti-PD-L1 d.

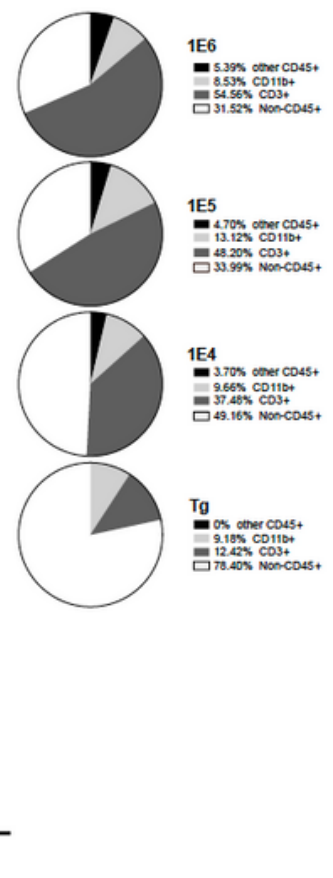

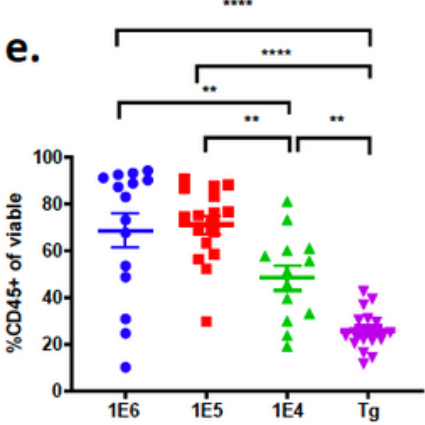
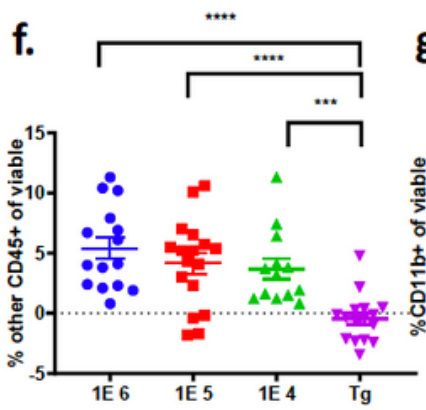

g.
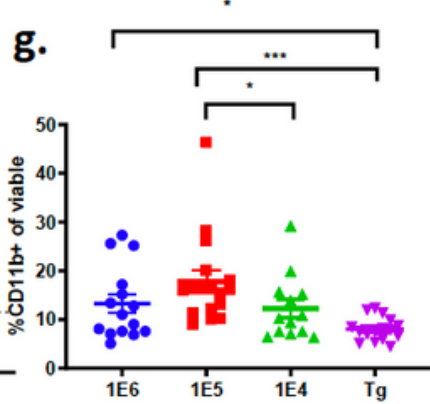

h

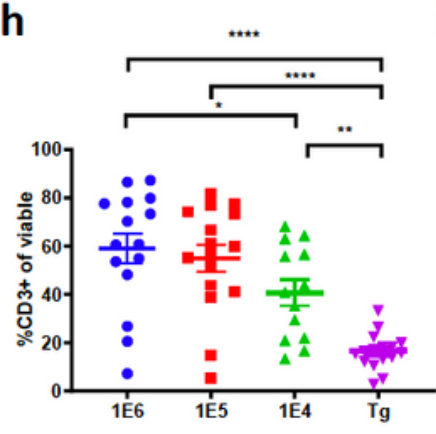

i.

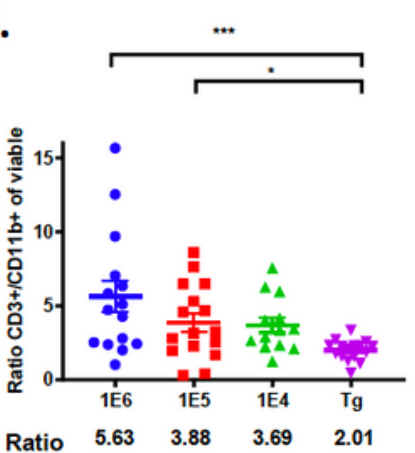

j.

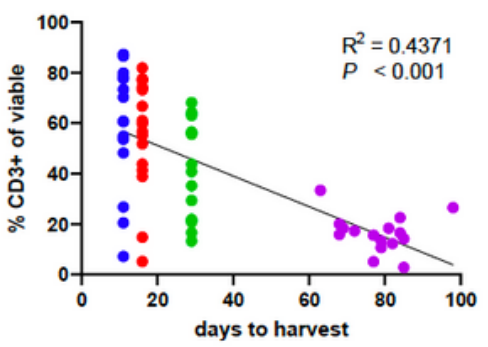

$1 E 6$

$1 E 5$

$1 \mathrm{E} 4$

Transgenic (Tg)

\section{Figure 1}

The number of cancer cells inoculated in preclinical models influences tumor growth kinetics as well as the tumor microenvironment. a. Tumors from the autochthonous MMTV-PyMT model were harvested and single cell suspensions were generated. Cells (1E6, $1 \mathrm{E} 5$ or 1E4) were injected into the mammary fat pad of FVB/NJ wild type mice. When the tumors reached $100 \mathrm{~mm} 3$, mice were randomized into an experimental group. b. Tumor inoculum was generated on three separate days and flow cytometry was 
performed to identify the cell proportions. c. Tumor volumes were measured every 3-4 days until individual tumors reached $100 \mathrm{~mm} 3$; tumor volumes are plotted as average tumor burden \pm SEM. $d$-j. Tumors were obtained when they reached $100 \mathrm{~mm} 3$ and flow cytometry was performed. $d$. Proportion of major cell types are shown. e. Percent of CD45+ cells out of total viable cells. f-h. Proportion of CD3+, $\mathrm{CD} 11 \mathrm{~b}+$, and other CD45+ cells of total viable cells. i. Ratio of CD3+ to CD11b+ out of viable cells. $j$. Regression analysis of the proportion of CD3+ out of viable cells, to time for tumor to reach $100 \mathrm{~mm} 3$. Graphs show mean \pm SEM from at least two independent experiments with at least three mice per group. Each data point represents an individual mouse. ${ }^{\star} P<0.05$, ${ }^{\star *} P<0.01, * \star \star P<0.001$ 
a. T-cells: of total (per gram of tumor tissue)
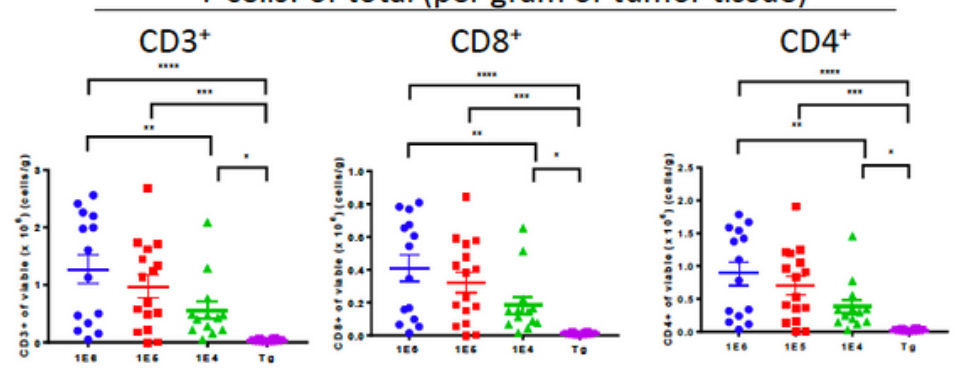

b.

T-cells: of $\mathrm{CD}^{4} 5^{+}$cells

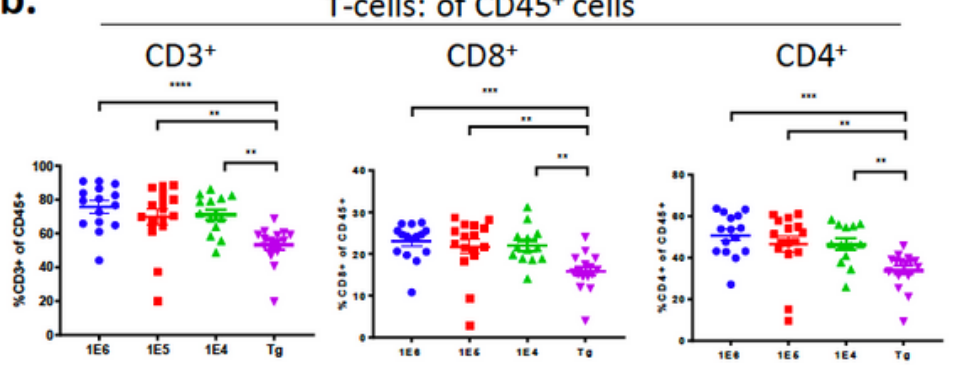

c.

Myeloid cells: of total (per gram of tumor tissue)

$\mathrm{F} 4 / 80^{+}$

$\mathrm{Gr} 1^{+} \mathrm{CD} 11 \mathrm{~b}^{+}$

$\mathrm{CD} 11 \mathrm{~b}^{+}$of $\mathrm{CD} 45^{+}$

$\mathrm{F} 4 / 80^{+}$of $\mathrm{CD} 45^{+}$

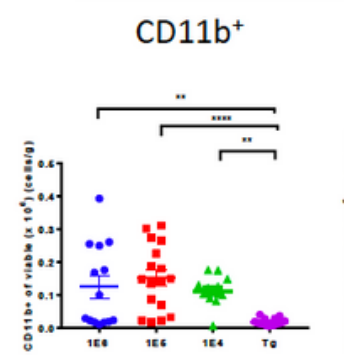

d.

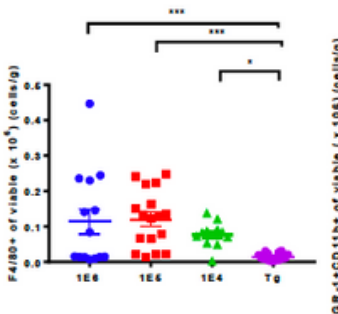

Myeloid cells: of $\mathrm{CD}^{4} 5^{+}$cells

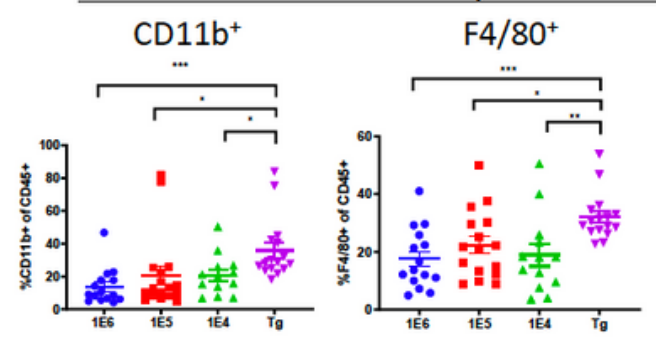

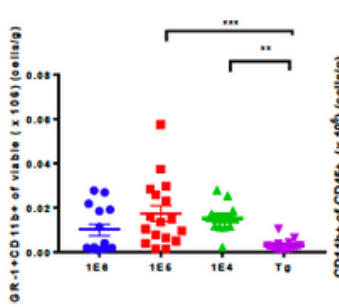

$\mathrm{Gr} 1^{+} \mathrm{CD} 11 \mathrm{~b}^{+}$

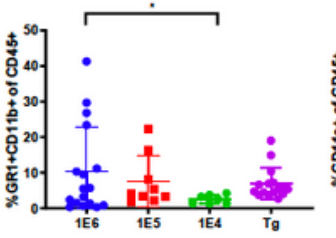

e.

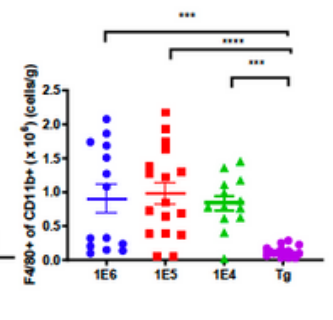

$\mathrm{CD} 11 \mathrm{~b} / \mathrm{CD} 3$
$\mathrm{CD}_{11 \mathrm{c}^{+}}$
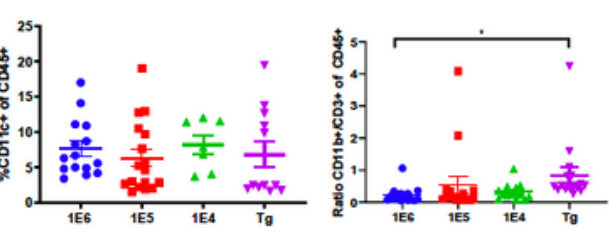

$1 \mathrm{E} 6$

$1 \mathrm{E5}$

$1 \mathrm{E} 4$

Transgenic (Tg)

\section{Figure 2}

Tumor infiltrating leukocyte populations differ significantly in the breast tumor models. Tumors from the autochthonous MMTV-PyMT model were harvested and single-cell suspensions were generated. Cells (1E6, $1 E 5$ or 1E4) were injected into the mammary fat pad of FVB/NJ wild type mice. When the tumors reached $100 \mathrm{~mm} 3$, tumors were obtained and processed into single-cell suspension for immunophenotyping by flow cytometry. a. Analysis comparing absolute number of lymphocytes (CD3+) 
and lymphocyte subsets (CD8+ and CD4+) per gram of tissue. b. Analysis comparing frequency of lymphocytes (CD3+) and lymphocyte subsets (CD4+ and CD8+) out of total CD45+ cells. c. Analysis comparing absolute number of myeloid cells $(\mathrm{CD} 11 \mathrm{~b}+)$ and myeloid cell subsets ( $\mathrm{F} 4 / 80+$ and $\mathrm{Gr} 1+\mathrm{CD} 11 \mathrm{~b}+)$ per gram of tissue. $d$. Analysis of myeloid cell subsets as a percent of total CD45+ cells. Graphs show mean \pm SEM from at least two independent experiments with at least three mice per group. Each data point represents an individual mouse. Unpaired two tailed t-test. ${ }^{*} P<0.05, * \star P<0.01, * \star \star$ $\mathrm{P}<0.001$, **** $\mathrm{P}<0.001$.
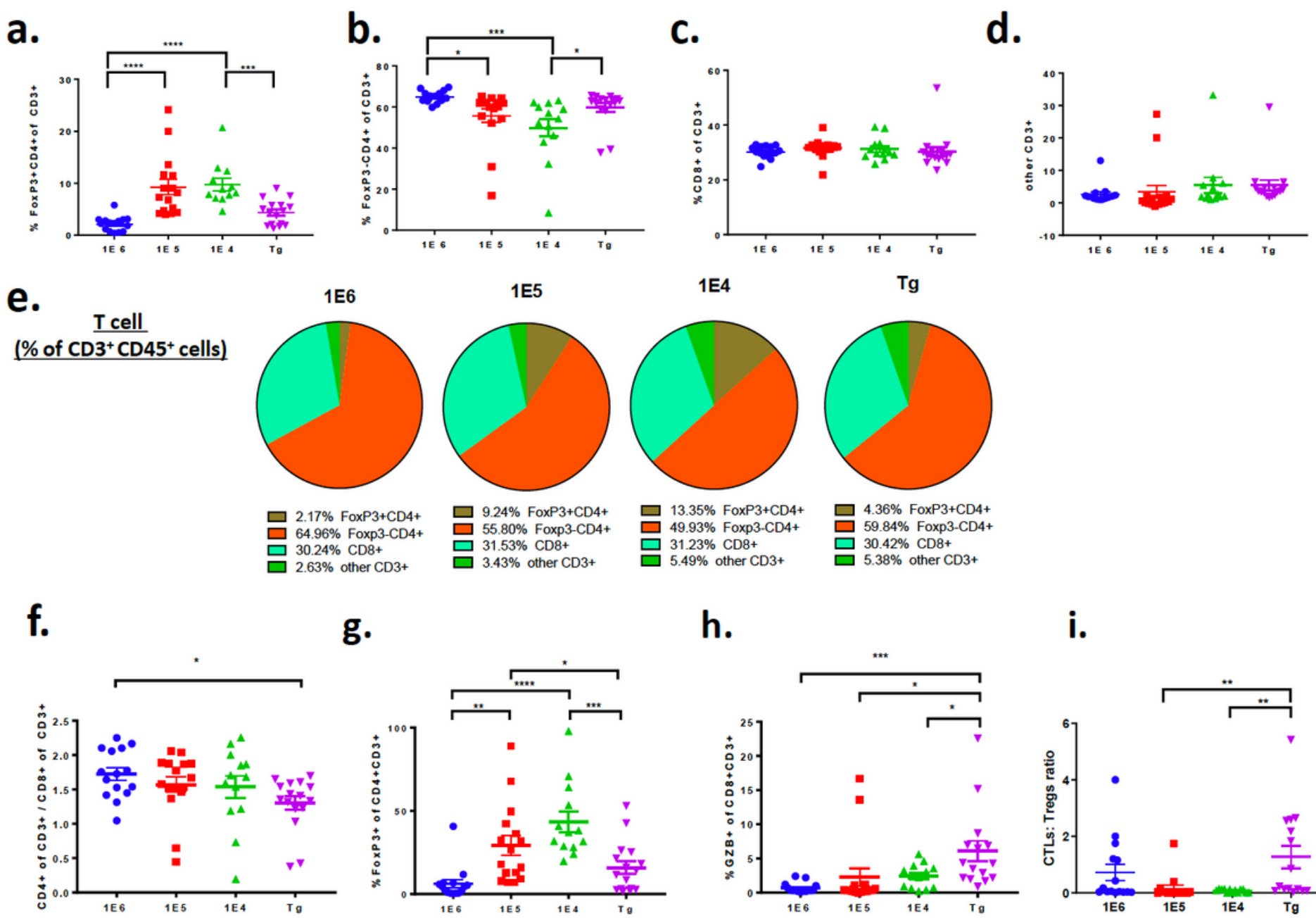

g.

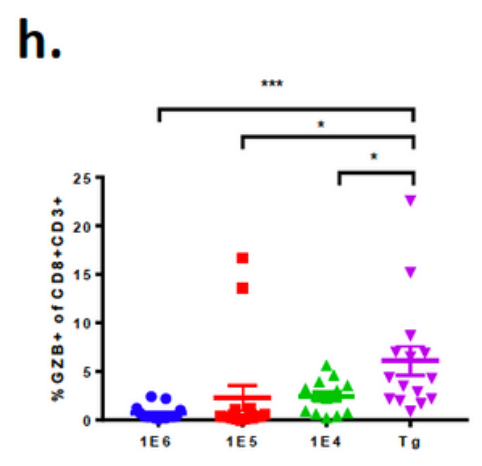

i.
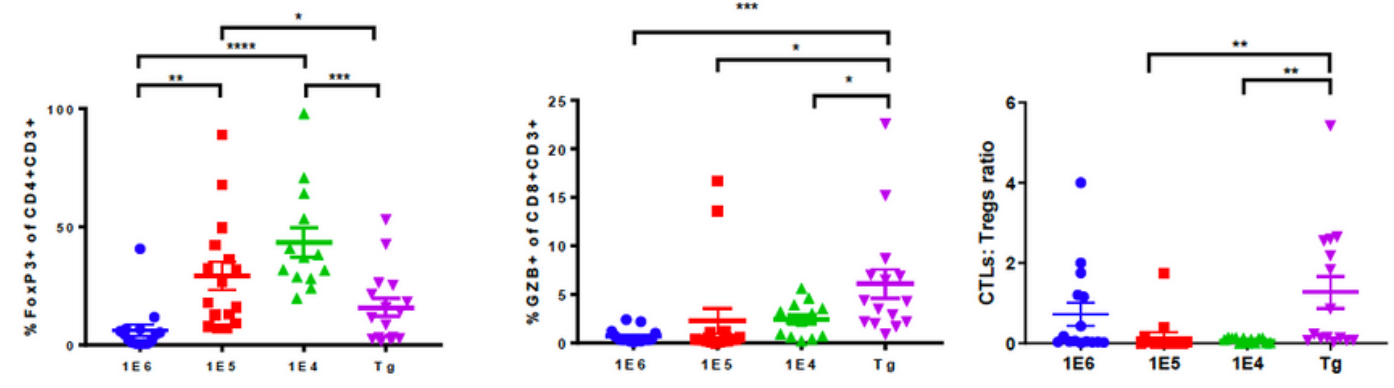

Figure 3

T-cell immune subsets differ significantly between the breast tumor models. Tumors from the autochthonous MMTV-PyMT model were harvested and single-cell suspensions were generated. Cells (1E6, 1E5 or 1E4) were injected into the mammary fat pad of FVB/NJ wild type mice. When the tumors reached $100 \mathrm{~mm} 3$, tumors were obtained and processed into single-cell suspension for immunophenotyping by flow cytometry. a-d. Analysis of T-cell subsets as a percent of CD3+ cells and include: a. T-regulatory cells (FoxP3+CD4+), b. FoxP3-CD4+, c. CD8+, and d. other CD3+ cells. e. Analysis of lymphocyte cell subsets as a percent of viable cells represented as pie charts. $f$. The ratio of CD4+ to CD8+ out of total lymphocytes. g. Analysis of T regulatory cells represented by FoxP3+ of CD4+ cells. h. The ratio of CTLs represented by GZB+ of CD8+. i. The ratio of CTLs to T-regulatory cells. Graphs show 
mean \pm SEM from at least two independent experiments with at least three mice per group. Each data

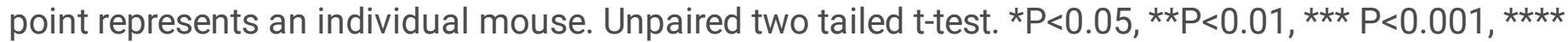
$P<0.001$.
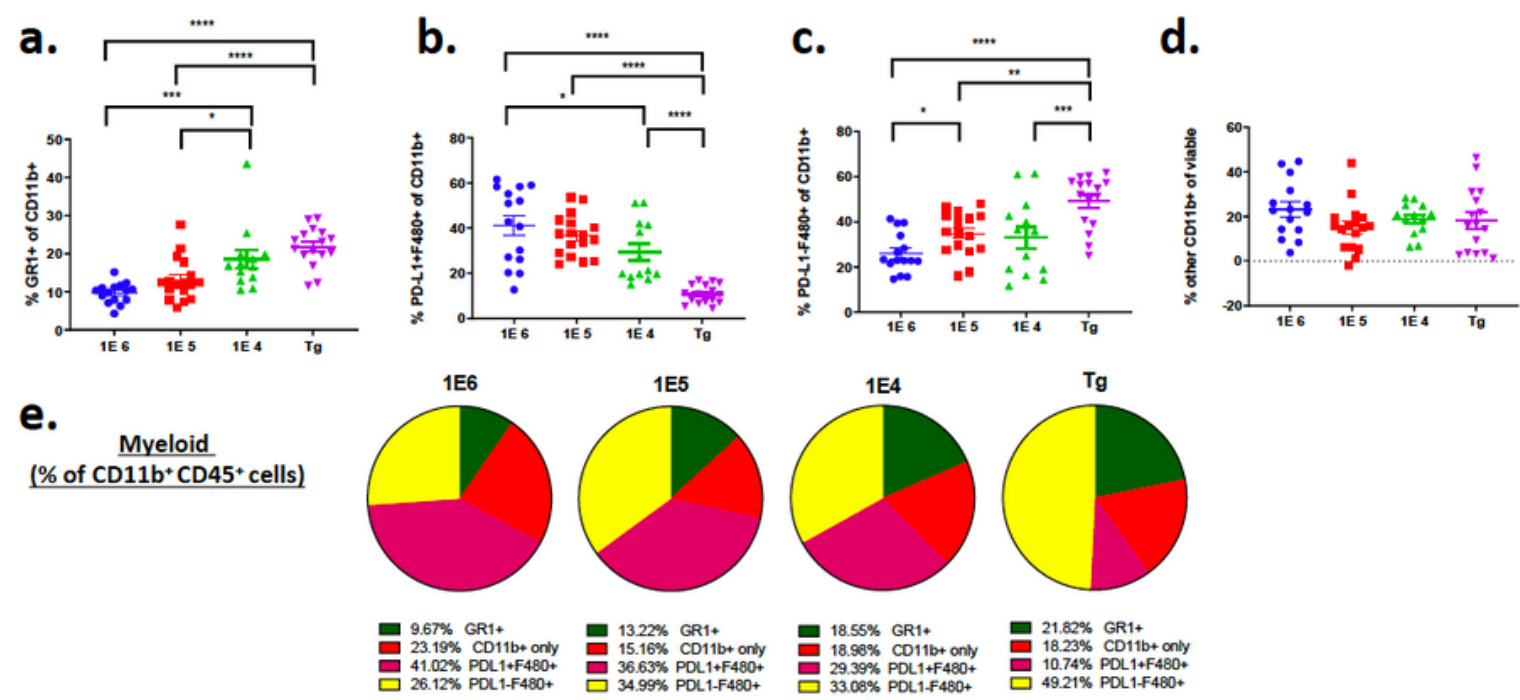

f.
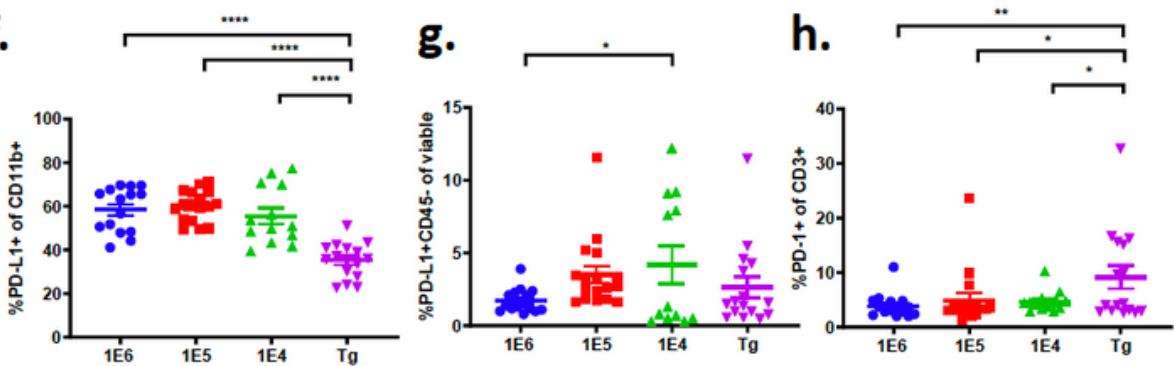

i.
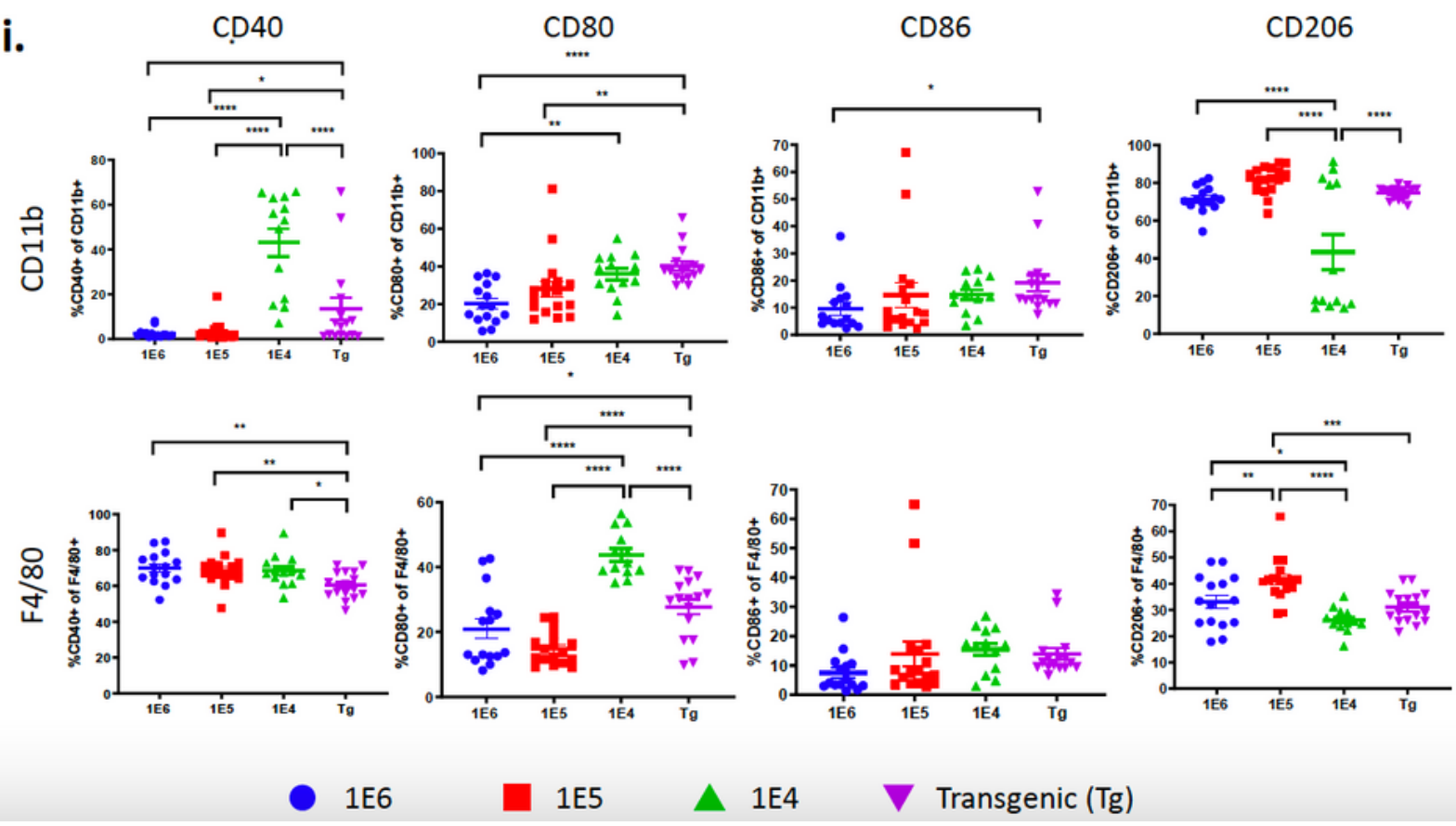

$1 \mathrm{E} 4$

Transgenic (Tg)

Figure 4

Myeloid immune cell subsets differ significantly breast tumor models. Tumors from the autochthonous MMTV-PyMT model were harvested and single-cell suspensions were generated. Cells (1E6, 1E5 or 1E4) 
were injected into the mammary fat pad of FVB/NJ wild type mice. When tumors reached $100 \mathrm{~mm} 3$, tumors were obtained and processed into single-cell suspension for immunophenotyping by flow cytometry. a-d. Analysis of cells subsets as a percent of myeloid cells (CD11b+) include a. myeloid derived suppressor cells (MDSC (GR1+)) b. PD-L1+F4/80+ C. PD-L1- F4/80+ d. other CD11b+ populations; e. Analysis of the myeloid cells subsets as a percent of viable cells include: $f$. Analysis of PD-L1 expression on myeloid cells (CD11b+) and g. cancer cells represented by CD45-. h. Analysis of the ratio of lymphocytes expressing PD-1. i. Analysis of M1-like macrophage markers, CD40 +, CD80 +, and CD86 + and an M2-like macrophage marker, CD206+, as a ratio of total myeloid cells (CD11b+, top), and mature macrophages (F4/80+, bottom). Graphs show mean \pm SEM from at least two independent experiments with at least three mice per group. Each data point represents an individual mouse. Unpaired

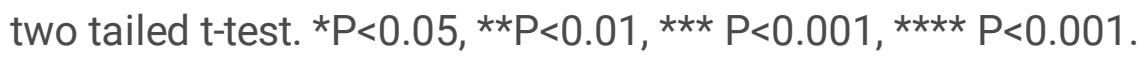


a.
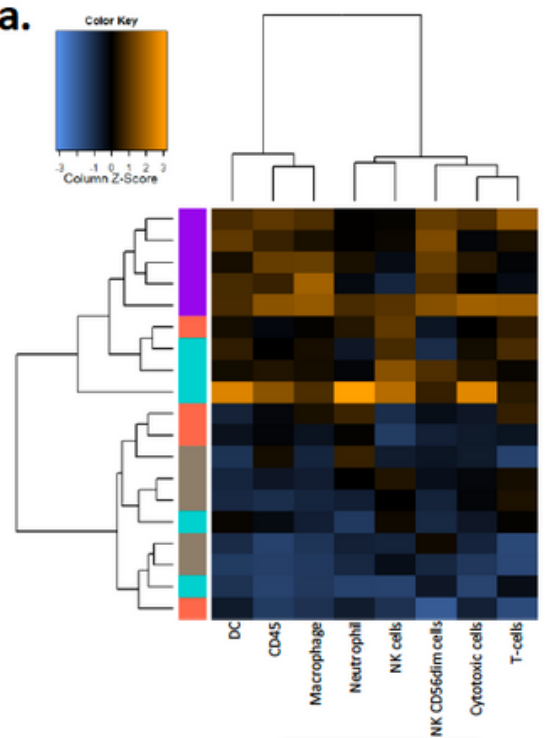

c.

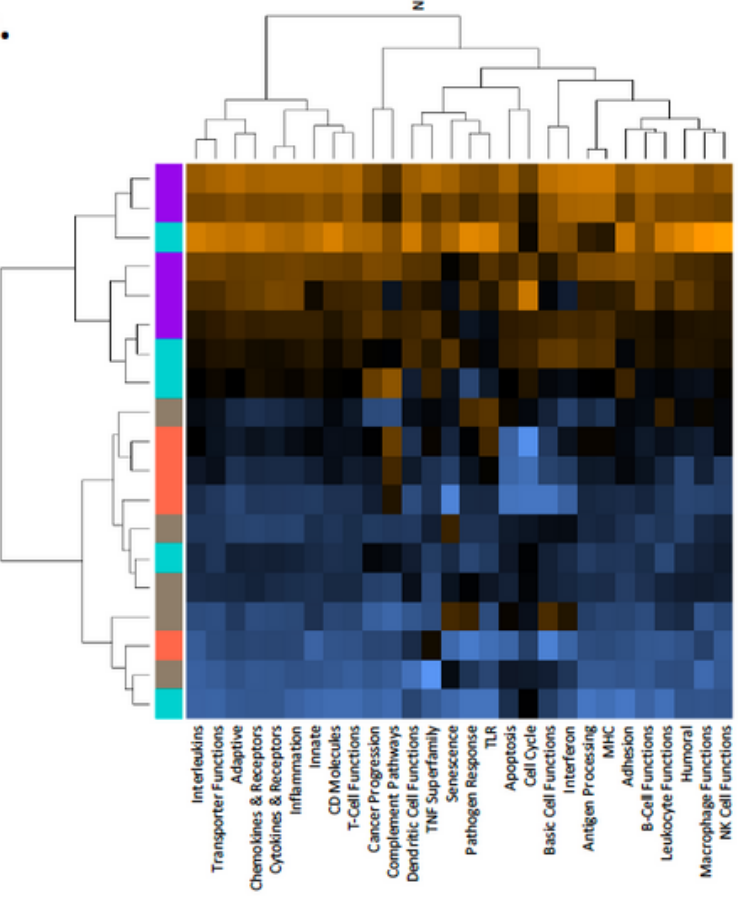

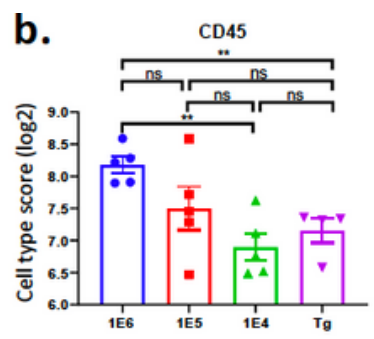
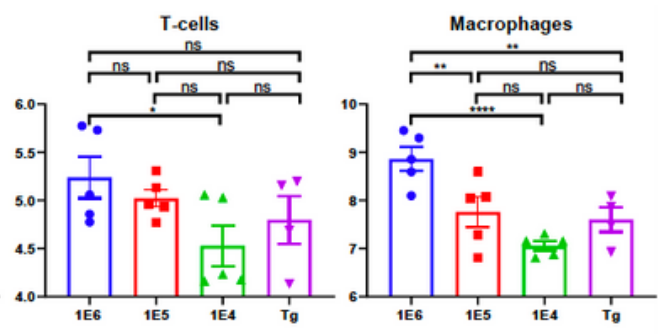

d.
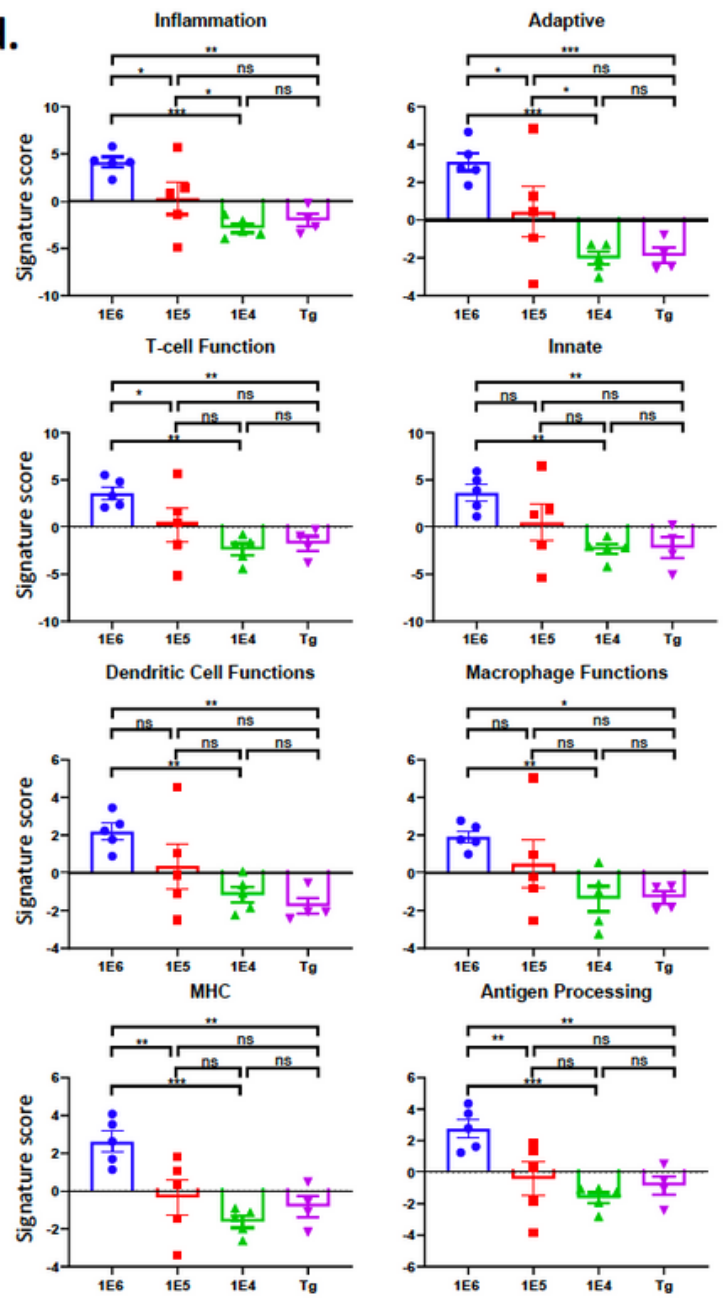
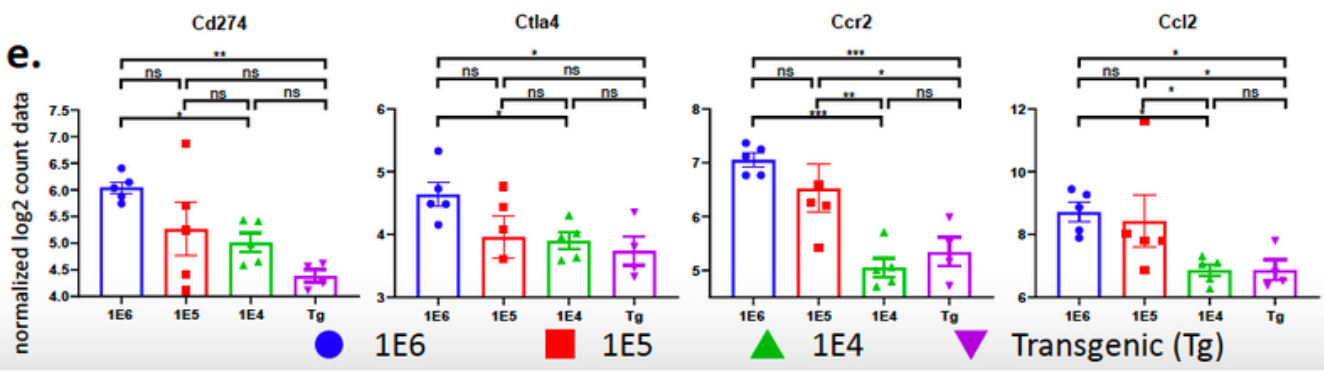

Figure 5

The breast tumor models have distinct immune transcriptional signatures. Tumors from the autochthonous MMTV-PyMT model were harvested and single-cell suspensions were generated. Cells (1E6, 1E5 or 1E4) were injected into the mammary fat pad of FVB/NJ wild type mice. When tumors reached $100 \mathrm{~mm}$, tumors were obtained, and RNA was extracted from tumors to assess transcriptional changes. The NanoString PanCancer Immune Profiling Panel was used to analyze gene expression 
patterns related to the immune cell compartment of the tumors. a. A heatmap of all cell-type scores are shown. b. Quantification of cell types scores of immune cells, T-cells, and macrophages are shown. c. Hierarchically clustered heatmap showing the pathway scores across the four models. Yellow and blue represent up and downregulated scores, respectively. $d$. Signature scores from the following pathways are highlighted: inflammation, adaptive immunity, T-cell function, innate immunity, dendritic cell functions, macrophage functions, $\mathrm{MHC}$, and antigen processing. e. Normalized log2 counts are shown for the following transcripts: Cd274 (PD-L1), Ctla4, Ccr2, and Ccl2. Graphs show mean \pm Standard Deviation with 4-5 mice per group. Each data point represents an individual mouse. Unpaired two tailed t-test. ${ }^{*}<<0.05$, $\star \star P<0.01, \star \star \star * P<0.001, \star \star \star \star ~ P<0.001$. 
a.
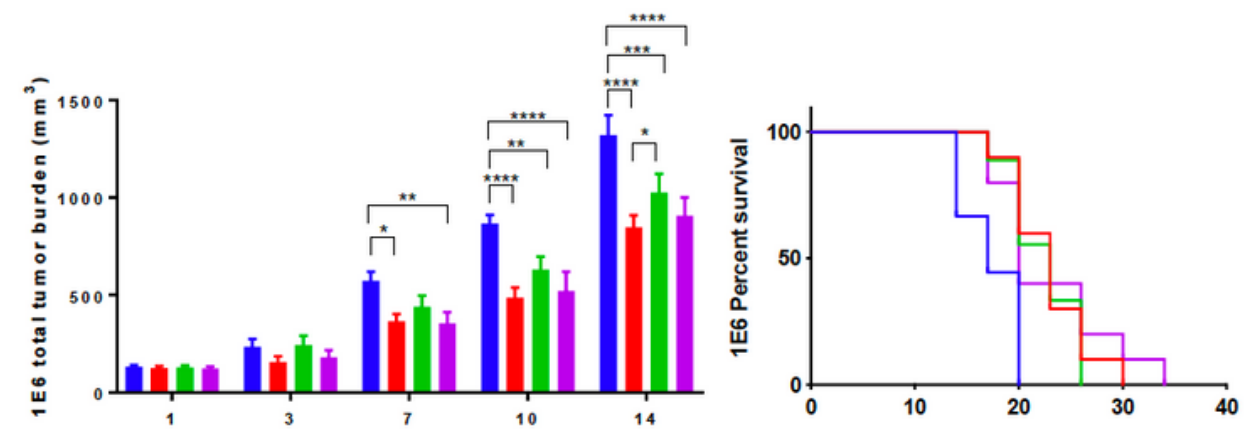

b.
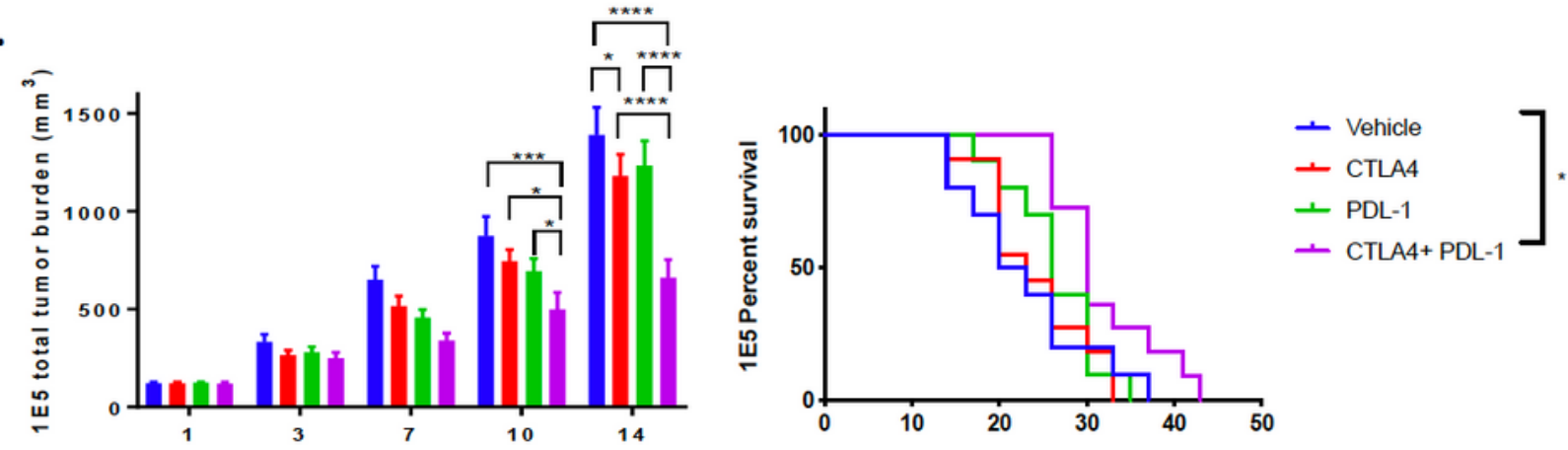

c.
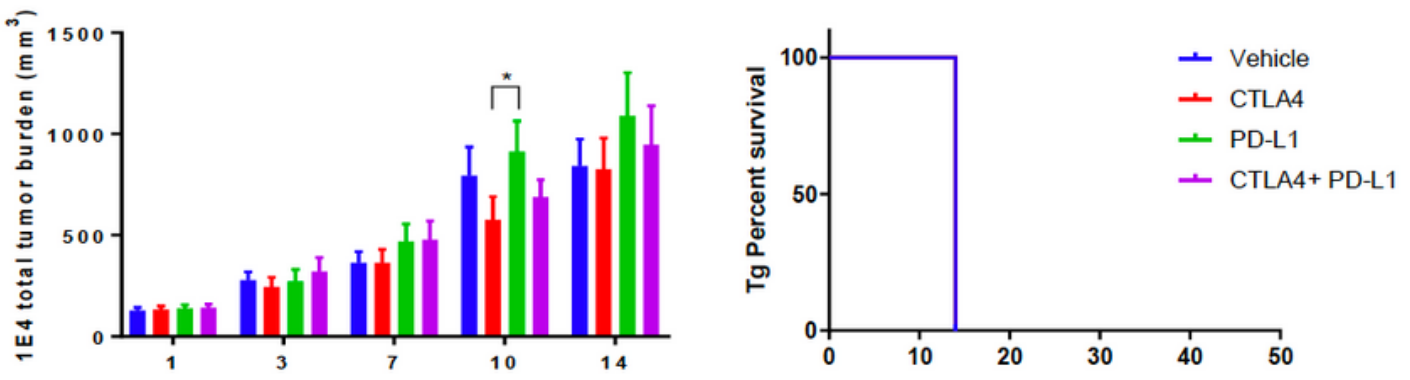

d.
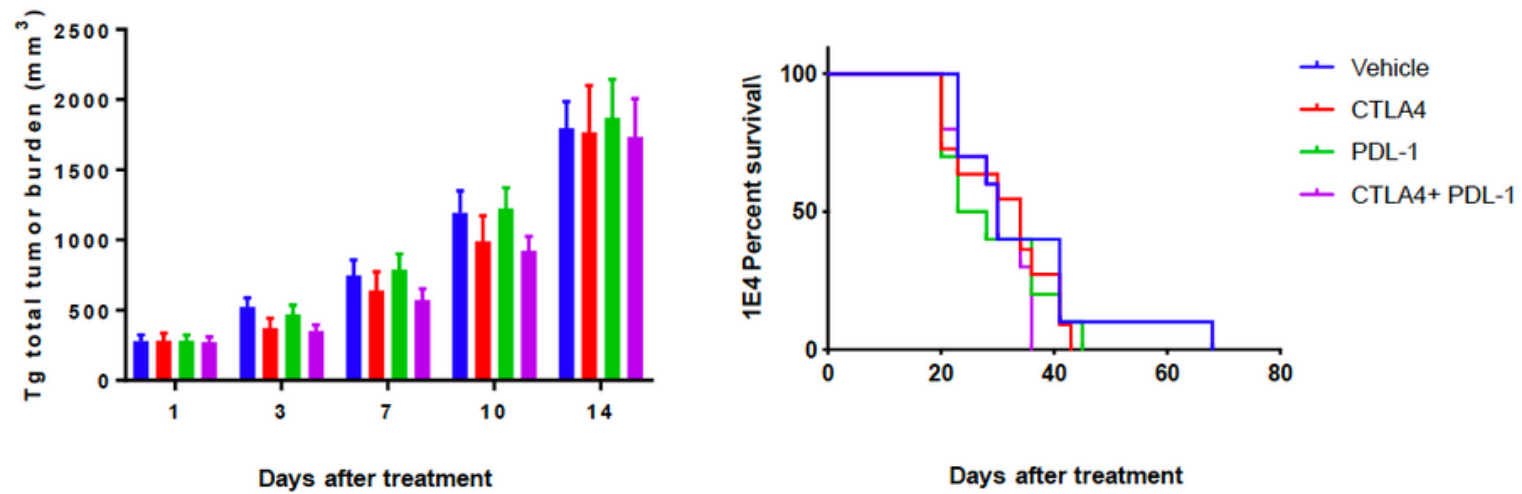

Vehicle $\square$ Anti-CTLA4 $\square$ Anti-PD-L1 $\square$ Anti-CTLA4 + Anti-PD-L1

\section{Figure 6}

The MMTV-PyMT syngeneic models respond differently to immune checkpoint blockade. Tumors from the autochthonous MMTV-PyMT model were harvested and single-cell suspensions were generated. Cells (1E6, 1E5 or 1E4) were injected into the mammary fat pad of FVB/NJ wild type mice. When the tumors reached $100 \mathrm{~mm} 3$, mice were randomized into four treatment groups: Vehicle (IgG2B), anti-PD-L1, antiCTLA-4, and the combination of anti-PD-L1 and anti-CTLA-4. Tumor volumes were measured and plotted 
as mean total tumor burden \pm SEM (left) and survival analysis of mice are shown (right). Shown is a representative experiment of two individual experiments $n=3-7 /$ treatment groups for the 1E6 (a), 1E5 (b), 1E4 (c) and transgenic models (d). In Fig. 6d all mice were sacrificed on the same day due to meeting endpoint criteria. Two-way ANOVA multiple comparisons and Gehan-Breslow-Wilcoxon tests were performed. ${ }^{*} \mathrm{P}<0.05, * * \mathrm{P}<0.01, * * * \mathrm{P}<0.001$, $* * * * \mathrm{P}<0.001$

a.

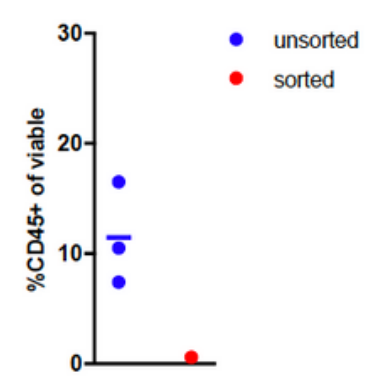

c.

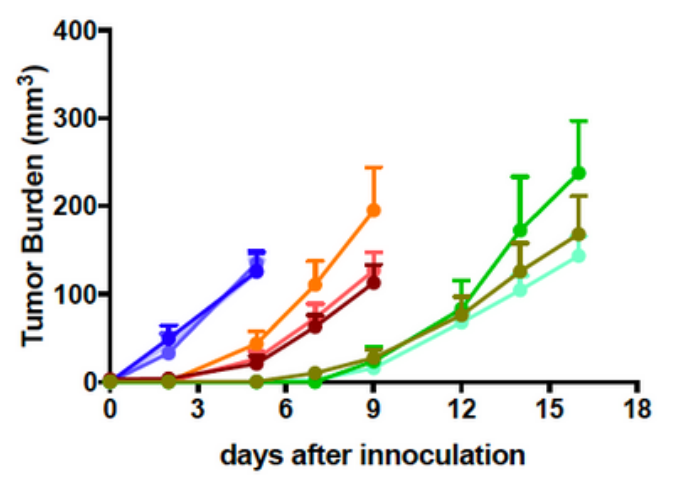

d.

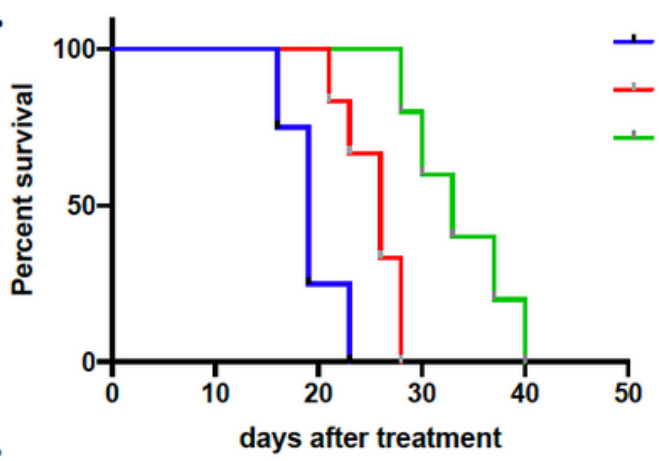

e.

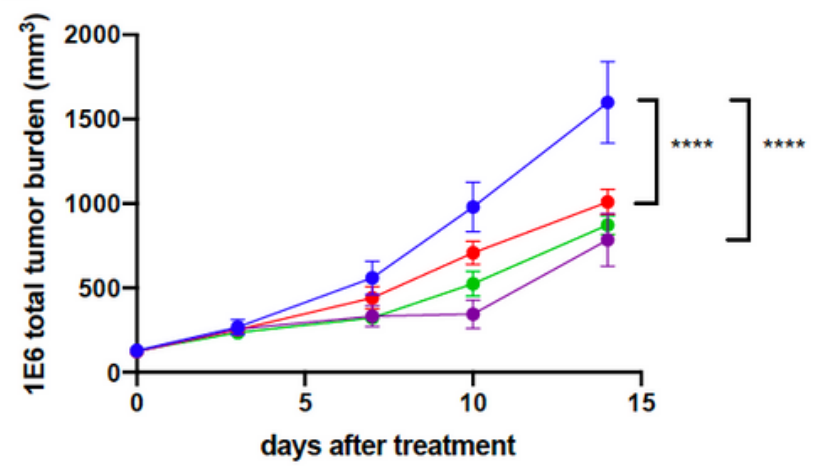

- Unsorted, Vehicle

$\rightarrow$ Unsorted, CTLA4+PD-L1

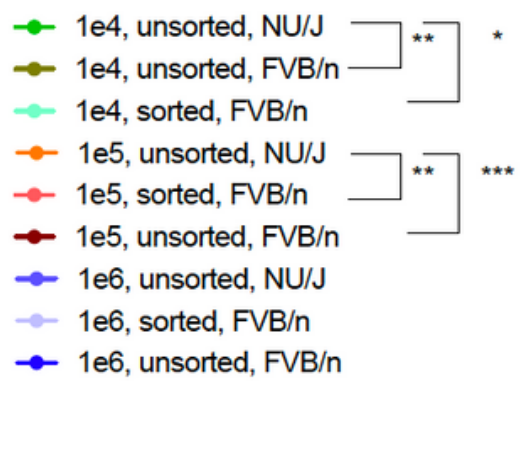

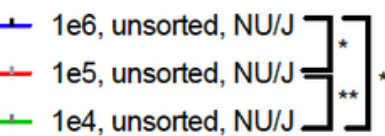

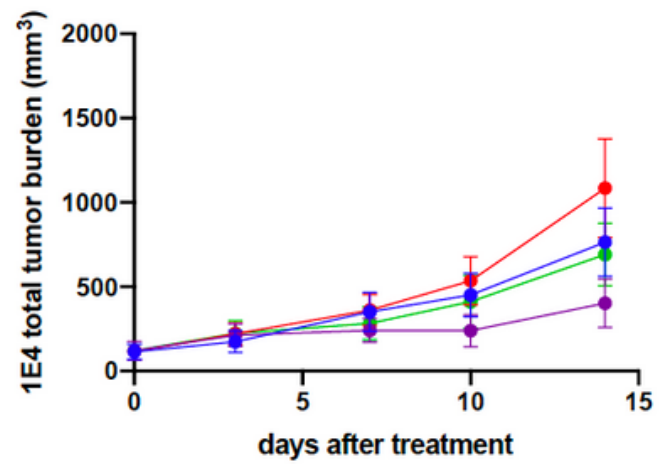

-- Sorted, Vehicle

- Sorted, CTLA4+PD-L1

Figure 7 
Testing tumor growth kinetics and immunotherapy response in immunodeficient murine models. Tumors from the autochthonous MMTV-PyMT model were harvested and single-cell suspensions were generated. CD45+ cells were successfully removed ("sorted") (a). b. Cells (1E6, 1E5 or 1E4) were injected into the mammary fat pad of FVB/NJ wild type or Nude mice. c. Tumor volumes were measured and plotted as mean total tumor burden \pm SEM and the survival of Nude mice are shown (d). e. When the tumors reached $100 \mathrm{~mm} 3$, mice were randomized to treatment with Vehicle (IgG2B) or the combination of antiPD-L1 and anti-CTLA-4. Tumor volumes were measured and plotted as mean total tumor burden \pm SEM.

\section{Supplementary Files}

This is a list of supplementary files associated with this preprint. Click to download.

- Tables.pdf

- SupplementalFigures.pdf 